\title{
LIFTING CYCLES TO DEFORMATIONS OF TWO-DIMENSIONAL PSEUDOCONVEX MANIFOLDS
}

\author{
BY
}

\author{
HENRY B. LAUFER ${ }^{1}$
}

\begin{abstract}
Let $M$ be a strictly pseudoconvex manifold with exceptional set $A$. Let $D>0$ be a cycle on $A$. Let $\omega$ : $\mathfrak{T} \rightarrow Q$ be a deformation of $M$. Kodaira's theory for deforming submanifolds of $\mathscr{T}$ is extended to the subspace $D$. Let $\mathcal{T}$ be the sheaf of germs of infinitesimal deformations of $D$. Suppose that $H^{1}(D, \mathcal{T})=0$. If $\omega$ is the versal deformation, then $D$ lifts to above a submanifold of $Q$. This lifting is a complete deformation of $D$ with a smooth generic fiber.

If all of the fibers of $T$ are isomorphic, then $\omega$ is the trivial deformation. If $M$ has no exceptional curves of the first kind, then there exists $\omega$ such that only any given irreducible component of $A$ disappears as part of the exceptional set.
\end{abstract}

I. Introduction. In [12], Kodaira develops a theory of deforming compact submanifolds within a given ambient manifold $\mathfrak{T}$. In this paper we develop an analogous theory for some singular subvarieties of $\mathfrak{R}$.

Let $M$ be a given strictly pseudoconvex two-dimensional manifold. Let $\omega$ : $\Re \rightarrow Q$ be a 1-convex deformation of $M$. (See $\S I I$ for the definition of 1-convex.) $M$ in fact has a versal deformation [19]. We are interested in how the exceptional set $A$ of $M$ changes under $\omega$. Essentially [18, §II, pp. 3-7] the new exceptional curves are liftings of cycles $D$ on the original exceptional set $A$. So it is important to see how cycles on $A$ lift to $Q$. Let $\Phi=\vartheta_{D}(D)$. Obstructions to lifting $D$ lie in $H^{1}(D, \Phi)$.

$\S \mathrm{II}$ is devoted to first obstructions. As an application, we prove the analogue of [5] and [25, Theorem 2.1 and Proposition 2.3]. (Theorem 2.11): If all of the fibers of a deformation $\omega: \mathfrak{N} \rightarrow Q$ of $M$ are isomorphic, then $\omega$ is the trivial deformation.

\$III is devoted to higher obstructions. We first do the formal theory. Convergence is then proved using Kodaira's methods, but there are some subtle points. Theorem 2.8 of [18] is proved here as Theorem 3.5. It says roughly the following: Suppose that $\omega$ is the versal deformation of $M$. Let $\mathcal{T}$ be the sheaf of germs of infinitesimal deformations of $D$. Suppose that $H^{1}(D, \mathcal{T})=0$. Then $D$ lifts precisely to a submanifold $S$ of $Q$ of codimension $\operatorname{dim} H^{1}(D, \Phi)$. There is an induced deformation $\lambda$ : $\mathscr{D} \rightarrow S$ of $D$ as an abstract analytic space. $\lambda$ is a complete deformation. The generic fiber of $\lambda$ is a manifold. We also show (Theorem 3.6) that if $M$ has no exceptional curves of the first kind, then $M$ may be deformed so that only any given irreducible component $A_{k}$ of $A$ disappears as part of the exceptional set.

Received by the editors May 27, 1980.

1980 Mathematics Subject Classification. Primary 32G05, 32G10; Secondary 14J15.

Key words and phrases. Deformation, pseudoconvex, exceptional set, obstruction.

${ }^{1}$ This research was partially supported by NSF Grant MCS 760496A01. 
Some of the results of this paper were obtained independently by Wahl [27], [28] in the algebraic category.

The author's manuscript [17] now essentially appears as three papers: [18], [19] and this paper.

II. First obstructions to lifting cycles. Recall that a map $\omega: \mathfrak{N} \rightarrow Q$ is 1 -convex if there is a real $C^{\infty}$ function $\varphi$ on $\mathscr{N}$ and a real number $c_{*}$ such that

(i) $\varphi$ is strictly plurisubharmonic on $\left\{m \in \mathscr{N} \mid \varphi(m)>c_{*}\right\}$ and

(ii) for all $c, \omega$ is a proper map on $\{m \in \mathfrak{T} \mid \varphi(m)<c\}$.

Let $\omega$ : $\mathfrak{N} \rightarrow Q$ be a 1-convex map which gives a deformation of the two-dimensional manifold $M=M_{0}=\omega^{-1}(0)$. Let $A=A_{0}$ be the exceptional set in $M$. Let $A_{q}$ be the exceptional set in $M_{q}=\omega^{-1}(q), q \in Q$. Let $W=\cup A_{q}$. Recall [23, p. 553] that $W$ is a subvariety of $\mathfrak{N}$. Let $V$ be an irreducible component of $W$ such that $A \cap V \neq \varnothing$. Let $P=\omega(V)$. Then by [18, Theorem 2.1, p. 3], there is a cycle $B=B_{0}$ on $A$ such that $V=\cup \operatorname{supp}\left(B_{q}\right), q \in P$, with $B_{q}$ the unique cycle on $A_{q}$ which is homologous to $B$ in $\mathfrak{T}$. We shall say that $B$ weakly lifts to $P$ locally. More generally and more formally,

DEFinition 2.1. Let $\omega: \mathfrak{N} \rightarrow Q$ be a 1-convex map which gives a deformation of the two-dimensional manifold $M=\omega^{-1}(0)$. Let $D=D_{0}>0$ be a cycle on $A$ and let $R$ be a reduced subvariety of $Q, 0 \in R$. D weakly lifts to $R$ locally if for each $r \in R_{1}$, some neighborhood of 0 in $R$, there is a (necessarily unique) cycle $D_{r}>0$ on $A_{r}$, the exceptional set $M_{r}=\omega^{-1}(r)$, such that $D$ and $D_{r}$ are homologous in $\Re$.

$D$ lifts to $R$ locally if there is a (nonreduced) subspace $\mathscr{D}$ of $\mathscr{T}$ such that $\lambda=\omega \mid \mathscr{D} \rightarrow R$ is a flat deformation of $D$ near 0 .

Of course, if $D$ lifts, then $D$ also weakly lifts. Namely, connect 0 and $r$ by a real curve $\gamma \subset R$. Then, suitably counting multiplicities, $\lambda^{-1}(\gamma)$ is a chain which shows that $D$ is homologous to $D_{r}$.

Recall [18, Proposition 2.7, p. 6],

Proposition 2.2. Let $\omega: \mathfrak{T} \rightarrow Q$ be a 1-convex map which gives a deformation of the two-dimensional manifold $M=\omega^{-1}(0)$. Let $D>0$ be a cycle on $A$, the exceptional set. Let $R=\left\{r \in Q \mid\right.$ there exists a cycle $D_{r}>0$ on $A_{r}$ such that $D_{r}$ is homologous to $D$ in $M$ \}. Then there is a sufficiently small neighborhood $Q_{1}$ of 0 in $Q$ such that $R_{1}=R \cap Q_{1}$ is a subvariety of $Q_{1}$. In particular, there is a maximal subvariety $R$ of $Q$ to which $D$ weakly lifts locally.

Let $S$ be any subvariety of $Q$ to which $D$ weakly lifts locally. Then $\cup D_{s}, s \in S$, is a subvariety of $\omega^{-1}(S)$. Let $\pi: T \rightarrow S$ be a resolution of $S$. Let $\tau: \Re \rightarrow T$ be the pull-back of $\omega$ via $\pi$. Then near any $t_{0} \in \tau^{-1}(0), D \subset M_{t_{0}}$ lifts to $T$ locally.

Proposition 2.2 implies that for $R$ smooth, the two notions of lifting in Definition 2.1 coincide. The author does not know if weak lifting always implies lifting in the case that $R$ is singular. However, in case $M$ is of dimension 3 and $A$ is of dimension 1, even for $R$ smooth, $A$ can weakly lift, but not lift; see Example 2.2, $k>2$, especially the end of $\S I I$, in [20].

We shall now examine how $D>0$ lifts by extending to the singular case some ideas of Kodaira. See [12], [1, Lemma 3.2, p. 339]. We shall use Kodaira's notation 
in [12] as much as possible. Until after Proposition 2.4 below, $M$ may be a manifold of arbitrary dimension and $D$ any compact divisorial cycle. $D$ need not lie on an exceptional set.

On $M, D$ locally has a single defining equation which may be reducible. Let $\mathfrak{U}=\left\{U_{i}\right\}$ be a cover of $M$ such that, on $U_{i}$, Id $D=\left(\tilde{w}_{i}\right)$. On $U_{i} \cap U_{j}$, there is a nonzero holomorphic function $\tilde{F}_{i j}$ such that

$$
\tilde{w}_{i}(z)=\tilde{F}_{i j}(z) \tilde{w}_{j}(z), \quad z \in U_{i} \cap U_{j} .
$$

On $U_{i} \cap U_{j} \cap U_{k}, \tilde{F}_{i j} \cdot \tilde{F}_{j k} \cdot \tilde{F}_{k i} \equiv 1$. Let $D$ also denote the nonreduced subspace of $M$ defined on each $U_{i}$ by $\left(D, \theta /\left(\tilde{w}_{i}\right)\right)$. Then the $\left\{\tilde{F}_{i j}\right\}$ give transition functions as follows for a line bundle $L$ over $D$. $L$ is trivial over $U_{i}$ with fiber coordinate $y_{i}$.

$$
y_{i}=\tilde{F}_{i j}(z) y_{j}, \quad z \in U_{i} \cap U_{j} .
$$

Let $\Phi$ be the sheaf of germs of sections of the line bundle $L$. Then $\Phi$ is canonically isomorphic to $\Theta_{D}(D)$ and also coincides with the normal sheaf to $D$ should $D$ be a submanifold.

Let $\omega: \Re \rightarrow Q$ be a deformation of $M=\omega^{-1}(0)$ with $Q$ smooth of dimension $d$. Deformations of the manifold $M$ are locally trivial. So taking $Q$ to be a small neighborhood of 0 and shrinking $M$, we may cover $\Re$ by the $U_{i} \times Q$.

We take the $U_{i}$ to be local coordinate patches on $M$ with local coordinate $z_{i}$. Then the change of coordinates on $\Re$ between $U_{j} \times Q$ and $U_{i} \times Q$ may be given by holomorphic maps $g_{i j}\left(z_{j}, q\right)$, i.e. $\left(z_{i}, q\right)=\left(g_{i j}\left(z_{j}, q\right), q\right)$. Let $f_{i j}\left(z_{j}\right)=g_{i j}\left(z_{j}, 0\right)$. Then $z_{i}=f_{i j}\left(z_{j}\right)$ is the change of coordinates between $U_{i}$ and $U_{j}$ as coordinate patches on $M$.

We shall use the nonsubscripted letter $z$ to denote a point in $M$. If also $z \in U_{i}$, then $z$ has coordinate $z_{i}$ on $U_{i}$. Recall from (2.1) the functions $\tilde{w}_{i}(z)$ on $U_{i}$ and $\tilde{F}_{i j}(z)$ on $U_{i} \cap U_{j}$. The product structure on $U_{i} \times Q$ allows an extension of $\tilde{w}_{i}$ from $U_{i} \times\{0\}$ to $U_{i} \times Q$. We denote this extension by $w_{i}\left(z_{i}\right)$. Note that $w_{i}\left(z_{i}\right)$ is strictly speaking a function both of $z_{i}$ and of $q \cdot w_{i}\left(z_{i}\right)=\tilde{w}_{i}(z)$. Now look at $\tilde{F}_{i j}(z)$, defined on $U_{i} \cap U_{j}$. We wish to extend $\tilde{F}_{i j}$ to $\left(U_{i} \times Q\right) \cap\left(U_{j} \times Q\right)$ as a subset of $U_{j} \times Q$. Initially, $\tilde{F}_{i j}$ only extends to $\left(U_{i} \cap U_{j}\right) \times Q$. However, this initial extension suffices for a shrunken $\left\{U_{i}\right\}$ and $Q$. So there is a holomorphic function $F_{i j}\left(z_{j}\right)$ on $\left(U_{i} \times Q\right) \cap\left(U_{j} \times Q\right) \subset U_{j} \times Q$ such that for $z \in U_{i} \cap U_{j}, F_{i j}\left(z_{j}\right)=\tilde{F}_{i j}(z)$. Again, $F_{i j}\left(z_{j}\right)$ is strictly speaking a function both of $z_{j}$ and of $q$. Observe that we do not claim that, for $q \neq 0$ and $z_{i}=g_{i j}\left(z_{j}, q\right), F_{i j}\left(z_{j}\right) F_{j i}\left(z_{i}\right) \equiv 1$. Let $T$ be smooth and $\pi$ : $T \rightarrow Q$ a holomorphic map, $\pi(0)=0$. Let $t=\left(t_{1}, \ldots, t_{n}\right)$ be local coordinates near 0 for $T$. In local coordinates, $q=\pi(t)=u\left(t_{1}, \ldots, t_{n}\right)$, where $u$ is $d$-tuple of holomorphic functions. The map $u$ will be important in the proof of Theorem 3.5 below. Let $\tau: \Re \rightarrow T$ be the pull-back of $\omega$ via $\pi$. Then $D$ lifts to $T$ if and only if for some cover $\mathfrak{U}=\left\{U_{i}\right\}$ of $M$, on each $U_{i} \times T$ there exists a holomorphic function $\varphi_{i}\left(z_{i}, t\right)$ and on each $\left(U_{i} \times T\right) \cap\left(U_{j} \times T\right)$ there exists a holomorphic function $h_{i j}\left(z_{j}, t\right)$ such that

$$
\begin{gathered}
\varphi_{i}\left(z_{i}, 0\right) \equiv 0, \quad h_{i j}\left(z_{j}, 0\right) \equiv 0, \quad \text { and with } z_{i}=g_{i j}\left(z_{j}, u(t)\right), \\
w_{i}\left(z_{i}\right)-\varphi_{i}\left(z_{i}, t\right)=\left[F_{i j}\left(z_{j}\right)+h_{i j}\left(z_{j}, t\right)\right]\left[w_{j}\left(z_{j}\right)-\varphi_{j}\left(z_{j}, t\right)\right] .
\end{gathered}
$$


Namely, if (2.3) is satisfied, let $\mathscr{D}$ be defined by $w_{i}\left(z_{i}\right)-\varphi_{i}\left(z_{i}, t\right)$ on $U_{i} \times T$. Conversely, the existence of the lifting $\mathscr{D}$ gives defining equation $w_{i}\left(z_{i}\right)-\varphi_{i}\left(z_{i}, t\right)$ on $U_{i}$.

We shall solve (2.3) via power series in $t$. Thus let

$$
\varphi_{i}\left(z_{i}, t\right)=\varphi_{i \mid 1}\left(z_{i}, t\right)+\cdots+\varphi_{i \mid p}\left(z_{i}, t\right)+\cdots
$$

with $\varphi_{i \mid p}\left(z_{i}, t\right)$ a homogeneous function of degree $p$ in $t$ with coefficients which are holomorphic functions of $z_{i}$. Let $h_{i j}\left(z_{j}, t\right)=h_{i j \mid 1}\left(z_{j}, t\right)+\cdots+h_{i j \mid p}\left(z_{j}, t\right)+\cdots$ with $h_{i j \mid p}\left(z_{j}, t\right)$ defined similarly. The coefficients of $h_{i j \mid p}$ are holomorphic on $U_{i} \cap U_{j}$. Letting $\equiv_{m}$ denote equality modulo $t^{m}$, to first order (2.3) is

$$
w_{i}\left(z_{i}\right)-\varphi_{i \mid 1}\left(z_{i}, t\right) \equiv_{2}\left[F_{i j}\left(z_{j}\right)+h_{i j \mid 1}\left(z_{j}, t\right)\right]\left[w_{j}\left(z_{j}\right)-\varphi_{j \mid 1}\left(z_{j}, t\right)\right] \text {. }
$$

To see if (2.4) can be satisfied, we shall transform the left side to $z_{j}$ coordinates, modulo $t^{2}$. Let $\rho_{0}$ : ${ }_{Q} T_{0} \rightarrow H^{1}(M, \Theta)$ be the Kodaira-Spencer map for $\omega$. It will suffice to identify $Q_{Q} T_{0}$ with $\{t\}$. Then, there is an $n$-tuple $\lambda_{i j}(z)$ of vector fields on $U_{i} \cap U_{j}$ such that $\rho_{0}(t)=\operatorname{cls}\left[t \cdot \lambda_{i j}\right]$ in $H^{1}(M, \Theta)$. Vector fields map functions to functions. $\lambda_{i j}(z)$ may also be thought of as a vector field depending on $z_{j}$ or on $z_{i}$. $\tilde{w}_{i}(z)$ may also be thought of as a function of $z_{i}$. Modulo $t^{2}$,

$$
\begin{aligned}
z_{i} & =g_{i j}\left(z_{j}, t\right) \equiv{ }_{2} f_{i j}\left(z_{j}\right)+\left(t \cdot \lambda_{i j} f_{i j}\right)\left(z_{j}\right), \\
w_{i}\left(z_{i}\right) & \equiv{ }_{2} \tilde{w}_{i}\left(f_{i j}\left(z_{j}\right)\right)+\left(t \cdot \lambda_{i j} \tilde{w}_{i}\right)\left(f_{i j}\left(z_{j}\right)\right) .
\end{aligned}
$$

Using (2.1), (2.4) becomes

$$
\begin{gathered}
\left(t \cdot \lambda_{i j} \tilde{w}_{i}\right)\left(z_{i}\right)-\varphi_{i \mid 1}\left(z_{i}, t\right)=-\tilde{F}_{i j}\left(z_{j}\right) \varphi_{j \mid 1}\left(z_{j}, t\right)+h_{i j \mid 1}\left(z_{j}, t\right) \tilde{w}_{j}\left(z_{j}\right) ; \\
z_{i}=f_{i j}\left(z_{j}\right) .
\end{gathered}
$$

The terms in (2.5) are all linear functions in $t$ and equality means that the coefficients are equal. Recall $\Phi$ of (2.2). Let $\Gamma\left(U_{i}, \Phi\right) t$ denote the group of linear functions in $t$ with coefficients in $\Gamma\left(U_{i}, \Phi\right)$. Recall that $\left(\tilde{w}_{i}\right)=\left(\tilde{w}_{j}\right)$ on $U_{i} \cap U_{j}$. Define $\psi_{i j}(z, t) \in \Gamma\left(U_{i} \cap U_{j}, \Phi\right) t$ by

$$
y_{i}=\psi_{i j}(z, t)=-\left(t \cdot \lambda_{i j} \tilde{w}_{i}\right)(z)=\left(t \cdot \lambda_{j i} \tilde{w}_{i}\right)(z), \bmod \left(\tilde{w}_{j}(z)\right) .
$$

$\left\{\psi_{i j}\right\}$ is in fact a cocycle. Indeed the cocycle condition is on $U_{i} \cap U_{j} \cap U_{k}$,

$$
\psi_{i k}(z, t)=\psi_{i j}(z, t)+\tilde{F}_{i j}(z) \psi_{j k}(z, t) .
$$

The cocycle condition is easily verified modulo Id $D$, or see (3.1) and its following below. The class of $\left[\psi_{i j}\right]$ is independent of choice of the cocycle $\left[\lambda_{i j}\right]$ in $\operatorname{cls}\left[\lambda_{i j}\right]$ in $H^{1}(M, \Theta)$.

Definition 2.3. For $t \in{ }_{Q} T_{0}, \delta(t)$, defined as the class of $\left[t \cdot \lambda_{j i} \tilde{w}_{i}\right]$ in $H^{1}(M, \Phi)$, is the first obstruction to lifting $D$ in the direction $t$.

Proposition 2.4. $D>0$ lifts infinitesimally to order 1 exactly on the subspace $S$ of ${ }_{Q} T_{0}$ where $\delta(t)=0$.

Proof. $D$ lifts to order 1 exactly if (2.5) is satisfied. $\varphi_{i \mid 1}$ and $\tilde{F}_{i j} \varphi_{j \mid 1}$ act as coboundaries, modulo $\tilde{w}_{j}$, in (2.5). So (2.5) can be satisfied if and only if $\delta(t)=0$.

The reason for the notation $\delta$ in Definition 2.3 is as follows. Using Kodaira's notation [12, p. 149], there is in the manifold case, and also in our codimension one 
case, an exact sequence over $D$

$$
0 \rightarrow \Phi \rightarrow \Psi \rightarrow \theta^{n} \rightarrow 0
$$

where $\Psi$ is the normal sheaf to $D$ in $\Re$. $\mathcal{O}^{n}$ corresponds to sections over $D$ of the pull-back to $D$ via $\omega$ of the tangent bundle $Q$ at 0 . Each $t \in{ }_{Q} T_{0}$ gives an element of $\Gamma\left(D, \theta^{n}\right) \approx \bigoplus \Gamma(D, \theta)$ which is a direct sum of constants. The coboundary map $\delta: \Gamma\left(D, \theta^{n}\right) \rightarrow H^{1}(D, \Phi)$ of (2.6) and $\delta$ of Definition 2.3 agree on $t$.

Proposition 2.5. Let $D>0$ be a divisor on an exceptional set $A$ in the two-dimensional manifold $M$. Let $\Phi=\mathcal{O}_{D}(D)$ from $(2.2)$. Then $\Gamma(M, \Phi)=0$.

Proof. The line bundle $L$ of which $\Phi$ is the sheaf of germs of sections restricts to a line bundle $L_{i}$ of Chern class $A_{i} \cdot D$ over an irreducible component $A_{i}$ of $A$ with $A_{i} \subset \operatorname{supp}(D)$. Let $\mathcal{E}_{i}$ be the sheaf of germs of sections of $L_{i}$. Since the intersection matrix $\left(A_{i} \cdot A_{j}\right)$ is negative definite, there is an $A_{1}$ such that $A_{1} \cdot D<0$. Let $G_{1}$ be the ideal sheaf of $A_{1}$ in $M$.

$$
0 \rightarrow \Phi_{1} \cdot \vartheta_{D}(D) \rightarrow \vartheta_{D}(D) \rightarrow \mathcal{L}_{1} \rightarrow 0
$$

is an exact sheaf sequence. $\Gamma\left(M, \mathscr{L}_{1}\right)=\Gamma\left(A_{1}, \mathscr{L}_{1}\right)=0$ since $L_{1}$ has negative Chern class. If $D=A_{1}$, then we are done. Otherwise, let $D^{\prime}=D-A_{1} \cdot \theta_{D}(D)$ is the sheaf of germs of meromorphic functions on $M$ which have poles at most $D$ modulo the sheaf of germs of holomorphic functions on $M$. Then, via the inclusion map on meromorphic functions, $\theta_{D^{\prime}}\left(D^{\prime}\right) \approx g_{1} \cdot \theta_{D}(D)$. Now use left exactness for sections of (2.7) and an induction argument to complete the proof.

We want to develop more information about the map $\delta$ of Definition 2.3. $\Phi$ is defined by the $\tilde{F}_{i j}$ of $(2.1)$, The following map $\beta: \Theta \rightarrow \Phi$ is easily seen to be a well-defined map of coherent sheaves of $\theta$-modules on $M$. If $\lambda$ is a vector field near a point $z_{0} \in M$ and $\tilde{w}(z)$ is a local defining equation for $D$ near $z_{0}$, then $\beta(\lambda)=(\lambda \tilde{w})(z) . \beta$ induces $\beta_{*}: H^{1}(M, \Theta) \rightarrow H^{1}(M, \Phi)=H^{1}(D, \Phi)$. Let $\rho_{0}: Q^{T} T_{0} \rightarrow$ $H^{1}(M, \Phi)$ be the Kodaira-Spencer map. Then, from Definition 2.3, $\delta=\beta_{*} \circ \rho_{0}$.

Let $A_{i}$ be an irreducible component of $A$. Let $\Phi_{i}$ be the $\Phi$ associated to $A_{i}$. As in the proof of Proposition 2.5, there is an inclusion map $\iota: \Phi_{i} \rightarrow \Phi$. The proof of the following lemma is straightfoward and will be omitted.

Lemma 2.6. Let $A_{i}$ be an irreducible component of the exceptional set $A$. Let $\boldsymbol{n}$ be the coefficient of $A_{i}$ in the cycle $D>0$. Then near any point which is in $A_{i}$ but is not in any other $A_{j}$ in the support of $D$, the two maps $\beta: \Theta \rightarrow \Phi$ and $\beta_{1}: \Theta \rightarrow \Phi_{1}$ satisfy $\beta=n \cdot\left(\iota \circ \beta_{1}\right)$.

Let $\mathcal{S}$ be the kernel of the map $\beta$. $\mathcal{S}$ is then the subsheaf of $\Theta$ of vector fields which take $D$ to $D$. See [26]. Note that $\mathcal{S}$, but not $\beta$, depends in fact only on $\operatorname{supp}(D)$, the support of $D$. Namely, near each regular point of $\operatorname{supp}(D)$, by Lemma 2.6, $\beta$ has the same kernel as some $\beta_{i}$. The regular points are dense in $\operatorname{supp}(D)$, so $\mathcal{S}$ is the intersection of the kernels of the $\beta_{i}$.

Let $\Re$ be the image of the map $\beta$. In case $\operatorname{supp}(D)$ has normal crossings, $\Re=\bigoplus \Re_{i}$, where $\Re_{i}$ is the sheaf of germs of sections of the normal bundle of $A_{i}$ in $M$, with the direct sum taken over $A_{i} \subset \operatorname{supp}(D)$ [26, Proposition 2.2, p. 333]. In 
any event, $0 \rightarrow \mathcal{S} \stackrel{\alpha}{\rightarrow} \Theta \stackrel{\gamma}{\rightarrow} \Re \rightarrow 0$ is an exact sequence. Since $\Re \subset \Phi$ and $\Gamma(M, \Phi)$ $=0$ by Proposition $2.5, \Gamma(M, \Re)=0$. So by [24]

$$
0 \rightarrow H^{1}(M, \delta) \stackrel{\alpha_{*}}{\rightarrow} H^{1}(M, \Theta) \stackrel{\gamma_{*}}{\rightarrow} H^{1}(M, \Re) \rightarrow 0
$$

is an exact sequence.

Let $\mathcal{T}$ be the sheaf of germs of infinitesimal deformations of $D$. See [8, p. 109]. $\mathcal{T}$ is defined locally in $z=(x, y)$ coordinates by $\theta /(\tilde{w}, \partial \tilde{w} / \partial x, \partial \tilde{w} / \partial y)$, where $\tilde{w}(z)$ is a local defining equation for $D$. Since $\beta$ maps $\partial / \partial x$ and $\partial / \partial y$ to $\partial \tilde{w} / \partial x$ and $\partial \tilde{w} / \partial y$

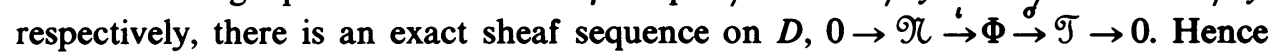
(2.9) below is also exact, using Proposition 2.5.

$$
0 \rightarrow \Gamma(D, \mathscr{T}) \rightarrow H^{1}(D, \mathscr{\Re}) \stackrel{\iota *}{\rightarrow} H^{1}(D, \Phi) \stackrel{\sigma_{*}}{\rightarrow} H^{1}(D, \mathscr{T}) \rightarrow 0 .
$$

Recall [19, Definition 1].

Definition 2.7. Let $M$ be a strictly pseudoconvex manifold. A special cover $\mathfrak{U}=\left\{U_{i}\right\}, 0<i<m$, is a finite cover of $M$ such that each $U_{i}$ is Stein and such that $\bar{U}_{i} \cap \bar{U}_{j} \cap \bar{U}_{k}=\varnothing$ for $1 \neq j \neq k$. ( - denotes closure in $M$.)

Obstructed cycles can now be seen to be most common, namely:

Theorem 2.8. Let $D>0$ be a cycle on the exceptional set $A$ in the strongly pseudoconvex two-dimensional manifold $M$. Suppose that no irreducible component $A_{i}$ in $\operatorname{supp}(D)$ is an exceptional curve of the first kind. Then $\beta_{*}: H^{1}(M, \Theta) \rightarrow$ $H^{1}(D, \Phi)$ is not the zero map.

Proof. Look at the proof of Proposition 2.5. For each $D^{\prime}$ (and $\left.\Phi^{\prime}\right), H^{1}\left(M, \Phi^{\prime}\right)$ is injected into $H^{1}(M, \Phi)$. Continue the argument until reaching the last $D^{\prime}$, which is an irreducible component $A_{1}$ of $\operatorname{supp}(D)$. Then $\iota_{*}: H^{1}\left(M, \Phi_{1}\right) \rightarrow H^{1}(M, \Phi)$ is an injection.

Since $A_{1}$ is reduced, the sheaf $\mathcal{T}_{1}$ of germs of infinitesimal deformations of $A_{1}$ is supported at only finitely many points. So $H^{1}\left(A_{1}, \mathcal{T}_{1}\right)=0$. So by (2.8) and (2.9), $\beta_{1, *}: H^{1}(M, \Theta) \rightarrow H^{1}\left(A_{1}, \Phi_{1}\right)$ is surjective. By Serre duality and the Riemann-Roch theorem,

$$
\operatorname{dim} H^{1}\left(A, \Phi_{1}\right)=g_{1}+\delta_{1}-A_{1} \cdot A_{1}-1,
$$

where $\delta_{1}$ is the "number" of nodes and cusps in the singular points of $A_{1}$. Since $A_{1}$ is, by the hypothesis, not an exceptional curve of the first kind, $H^{1}\left(A_{1}, \Phi_{1}\right) \neq 0$.

We can find a nonzero element in the image of $\beta_{*}$ as follows. Restriction is an isomorphism on $H^{1}(M, \Theta)$ [14, Lemma 3.1, p. 599]. So by [19, Theorem 2], we may assume that $M$ has a special cover $\mathfrak{U}=\left\{U_{i}\right\}$. Also, the construction of [19, Theorem 2] allows us to assume that each singular point of $A$ is an element of only one of the $U_{i}$. On $N(\mathfrak{u})$, take a nonzero element of $H^{1}\left(A_{1}, \Phi_{1}\right)$ as the image of a 1-cocycle $c$ from $\Theta$. Change the cocycle $c$ to be 0 on the $U_{i} \cap U_{j}$ which do not meet $A_{1} \cdot \beta_{1}(c)$ is unchanged and still nonzero. Since $\iota_{*}$ is an inclusion, by Lemma 2.6, $\beta(c)$ is also nonzero.

Let ${ }_{D} \Theta$ be the sheaf of germs of vector fields on $D$. If $\mathfrak{n}$ denotes the ideal sheaf of $D,{ }_{D} \Theta$ is the tangent sheaf ${ }_{n} \Theta$ of Grauert [6, p. 357]. ${ }_{D} \Theta$ is denoted by ${ }_{D} \theta$ in [15] and by just $\Theta$ in [16]. Let $\mathscr{G}$, rather than $\mathfrak{n}$, denote the ideal sheaf of $D$ in $M$. Then also ${ }_{D} \Theta=\mathcal{S} / \mathscr{S} \mathcal{S}$. 
LemMa 2.9. Let $M$ be a strictly pseudoconvex two-dimensional manifold. Then the map $\tau: H^{1}(M, \delta) \rightarrow H^{1}\left(M,{ }_{D} \Theta\right)$ is surjective for all cycles $D$ on the exceptional set. For all sufficiently large cycles $D, \tau$ is an isomorphism.

Proof. $H^{1}(M, \mathcal{S}) \stackrel{\sigma}{\rightarrow} H^{1}(M, \delta) \rightarrow H^{1}\left(M,{ }_{D} \Theta\right) \rightarrow 0$ is exact since $H^{2}(M, \mathcal{S} \delta)$ $=0$. By [6, Satz 1, p. 355], $\sigma$ is the zero map for all sufficiently large $D$.

Proposition 2.10. Let $\omega: \Re \rightarrow Q$ be a deformation of the strictly pseudoconvex two-manifold $M=\omega^{-1}(0)$ such that $\rho_{0}:{ }_{Q} T_{0} \rightarrow H^{1}(M, \Theta)$ is surjective. Let $D$ be a cycle on the exceptional set of $M$. Let $S$ be the subspace of Proposition 2.4. Then the infinitesimal lifting of $D$ to $S$ is an infinitesimally complete deformation of $D$.

Proof. By (2.8) and (2.9), $H^{1}(M, \Theta)$ maps onto $\Gamma(D, \mathcal{T})$. By Lemma 2.9, $H^{1}(M, \mathcal{S})$, a subspace of $H^{1}(M, \Theta)$, maps onto $H^{1}\left(D,{ }_{D} \Theta\right)$. The lemma now follows from the description [8] of the infinitesimal deformations of $D$.

In the case of normal crossings, $H^{1}(M, \Theta)$ can often be conveniently computed by letting $D$ be the reduced exceptional set. Since $\Re=\bigoplus \Re_{i}, H^{1}(M, \Re)$ in (2.8) is easily computed by Serre duality and the Riemann-Roch theorem. $H^{1}(M, \mathcal{\delta}) \approx$ $H^{1}\left(M,{ }_{D} \Theta\right)=H^{1}\left(D,{ }_{D} \Theta\right)$ for large $D$ by Lemma 2.8. $H^{1}\left(D,{ }_{D} \Theta\right)$ can often be computed as in [16].

THEOREM 2.11. Let $M$ be a strictly pseudoconvex manifold of dimension two. Let $A$ be the exceptional set of $M$. Let $\pi:$ T $\rightarrow P$ be a deformation of $M=\pi^{-1}(0)$ such that $P$ is reduced, $\pi$ is 1-convex and for each $p \in P$, some neighborhood of $A_{p}$ in $M_{p}=\pi^{-1}(p)$ is biholomorphic to some neighborhood of $A$ in $M$. Then given any compact set $K \subset M, \pi$ is the trivial deformation near $K$ in $\Re$.

Proof. Let $\omega: \Re \rightarrow Q$ be the versal deformation of $M$ [19, Theorem 8]. Let $f$ : $P_{1} \rightarrow Q_{1}$, with $P_{1}$ and $Q_{1}$ neighborhoods of 0 in $P$ and $Q$, respectively, induce $\pi$ from $\omega$ near $K$. We want that $f\left(P_{1}\right)=0$. Since some neighborhood of $A_{p}$ is biholomorphic to some neighborhood of $A, \rho_{q}: Q_{Q} T_{q} \rightarrow H^{1}\left(M_{q}, \Theta\right)$ is an isomorphism for any $q \in f\left(P_{1}\right)$. Thus it suffices to prove Theorem 2.11 for smooth $P$. For on the regular points of $P_{1}$, df will then be 0 . Then $f$ is a constant map on $P_{1}$. Hence $f\left(P_{1}\right)=0$ as desired.

We now assume that $P$ is smooth. By [18, Proposition 2.3, p. 4] every irreducible component $C_{i p}$ of $A_{p}$, for small $p$, is homologous to an integral cycle $\sum a_{i j} A_{j}$, $a_{i j}>0$, on $A$; we shall write $C_{i, p} \sim \sum a_{i j} A_{j}$. By hypothesis, the $C_{i, p}$ have an intersection matrix isomorphic to $\left(A_{i} \cdot A_{j}\right)$. It then follows that $a_{i j}=0$ for all $j$ except for one $j_{0}$ and $a_{i j_{0}}=1$. For if not, choosing subscripts so that the isomorphism has $C_{i, p} \approx A_{i}$, form $D_{i}=\sum a_{i j} C_{j, p} \sim \sum a_{i j}\left(\sum a_{j k} A_{k}\right) .\left(D_{i} \cdot D_{j}\right) \approx\left(A_{i} \cdot A_{j}\right)$. Iterating this process would give cycles $E_{i}$ on $A$, some of whose coefficients are arbitrarily large, with the same intersection matrix as $\left(A_{i} \cdot A_{j}\right)$. But then $\left(\Sigma E_{i}\right) \cdot$ $\left(\sum E_{i}\right)$ is both arbitrarily large and equal to $\left(\sum A_{i}\right) \cdot\left(\sum A_{i}\right)$, a contradiction. Then each $A_{i}$ lifts weakly to $P$ locally. By Proposition 2.2 , each $A_{i}$ lifts to $P$ locally.

Multiplying the equations for the deformations of the $A_{i}$ gives a deformation $\lambda$ of $A$. Let $\mu(s)$ be the Milnor number of a singular point $s \in A$. Under deformation, each $\mu(s)$ is upper-semicontinuous. Since $A \approx A_{p}$, each $\mu(s)$ is constant for small $p$ 
and the deformation of $A$ by $\lambda$ is equisingular [21]. So we may simultaneously blow up at the singular points of $A_{p}$ and still satisfy the hypothesis of this theorem. In particular, we may assume without loss of generality that $A$ has nonsingular irreducible components and normal crossings. Then, also, multiplying the equations for the deformations of the $A_{i}$ gives a locally trivial deformation of any cycle $D$ on $A$. Choose $D$ to have equal coefficients for all $A_{i}$ and sufficiently large so that $\tau$ of Lemma 2.9 is an isomorphism.

Let $f: P_{1} \rightarrow Q_{1}$ be as in the first paragraph of this proof. Then by [15, Theorem 3.6, p. 76] $d f(p)=0$ for all $p \in P_{1}$. Hence $f \equiv 0$, as required to complete the proof of the theorem.

Recall that any isolated singularity $p \in V$ has a versal deformation [7], [22], [11], [4]. If there are several isolated singularities, we may form the Cartesian product of the parameter spaces to get a versal deformation.

Corollary 2.12 [2], [1]. Let $\omega: \mathfrak{T} \rightarrow Q$ be a 1-convex map which is the versal deformation of the two-dimensional strictly peudoconvex manifold $M=\omega^{-1}(0)$. Suppose that $M$ has no exceptional curves of the first kind. Let $\pi: M \rightarrow V$ be the blow-down of $M$. Let $T$ be the simultaneous-blow-down subspace of $Q$. Let $\pi_{a}$ : $\mathcal{X} \rightarrow T$ be the blow-down of $\omega$ over $T$. Let $\tau: \mathcal{T} \rightarrow U$ be the versal deformation of the singularities of $V$. Let $f: T_{1} \rightarrow U_{1}$ be a holomorphic map which induces $\pi_{a}$ from $\tau$ near the singularities of $V ; T_{1}$ and $U_{1}$ are neighborhoods of 0 in $T$ and $U$ respectively. Then there are arbitrarily small $T_{1}$ and $U_{1}$ for which $f$ is finite and proper.

Proof. By Theorem 2.11, for small $T_{1}, f^{-1}(0)=0$. The corollary now follows as below. We first reduce to the case where $T$ and $U$ are manifolds of the same dimension. $f$ is induced near $0 \in T$ by an ambient map $g: \Delta \rightarrow U$ with $\Delta$ a polydisc. $g^{-1}(0) \cap T=0$. Let $D$ be an ambient neighborhood of 0 in $U \cdot g=$ $\left(g_{1}, \ldots, g_{n}\right): \Delta \rightarrow D$. There are additional holomorphic functions $g_{n+1}, \ldots, g_{m}$ on $\Delta$ such that $g_{i}(T)=0, n+1 \leqslant i \leqslant m$, and $\left\{g_{1}=\cdots=g_{m}=0\right\}=0$. Note that $\left\{g_{1}, \ldots, g_{m}\right\}$ induces the map $f$. Let $r$ be the dimension of $\Delta$. Then there are $r$ linear combinations of the $g_{i}, 1<i<m$, whose common zeroes $W$ have codimension $r$. Then there is a smaller $\Delta^{\prime}$ with $W \cap \Delta^{\prime}=0$. Using these $r$ linear combinations, we may assume that $T$ and $U$ are equidimensional manifolds.

Let $K$ be a small sphere about 0 in $T$. Let $\nu \geqslant 1$ be the multiplicity of the map $f$ : $K \rightarrow U-\{0\}$. Let $U_{1}$ be a connected neighborhood of 0 in $U$ with $U_{1} \cap f(K)=$ $\varnothing$. Let $B$ be the ball in $T$ having $K$ as its boundary. Let $T_{1}=f^{-1}\left(U_{1}\right) \cap B$. Then $f$ : $T_{1} \rightarrow U_{1}$ is a $\nu$-fold branched covering map; for $t \in T_{1}$, let the multiplicity be the degree of $f$ on a small sphere about $t . f: T_{1} \rightarrow U_{1}$ is necessarily proper. This completes the proof of Corollary 2.12 .

III. Higher order obstructions. Let us now return to solving (2.3). In order for $D$ to lift to $T$, by Proposition 2.4, the tangential map for $u$ must map to the kernel of $\delta: \quad Q^{T_{0}} \rightarrow H^{1}(D, \Phi)$. Suppose that (2.3) is satisfied modulo $t^{m+1}$, i.e. let $u^{m}(t)=$ $u_{1}(t)+\cdots+u_{p}(t)+\cdots+u_{m}(t)$ where $u_{p}(t)$ is a homogeneous polynomial of order $p$ in $t$. 


$$
\begin{aligned}
& \varphi_{i}^{m}\left(z_{i}, t\right)=\varphi_{i \mid 1}\left(z_{i}, t\right)+\cdots+\varphi_{i \mid p}\left(z_{i}, t\right)+\cdots+\varphi_{i \mid m}\left(z_{i}, t\right), \\
& h_{i j}^{m}\left(z_{j}, t\right)=h_{i j \mid 1}\left(z_{j}, t\right)+\cdots+h_{i j \mid p}\left(z_{j}, t\right)+\cdots+h_{i j \mid m}\left(z_{j}, t\right) .
\end{aligned}
$$

Let $\equiv_{m+1}$ denote equality modulo $t^{m+1}$. Then we are supposing that for $z_{i}=$ $g_{i j}\left(z_{j}, u^{m}(t)\right), z_{j} \in U_{i} \cap U_{j}$,

$$
w_{i}\left(z_{i}\right)-\varphi_{i}^{m}\left(z_{i}, t\right) \equiv_{m+1}\left[F_{i j}\left(z_{j}\right)+h_{i j}^{m}\left(z_{j}, t\right)\right]\left[w_{j}\left(z_{j}\right)-\varphi_{j}^{m}\left(z_{j}, t\right)\right] .
$$

We want to find $u_{m+1}(t), \varphi_{i \mid m+1}\left(z_{i}, t\right)$ and $h_{i j \mid m+1}\left(z_{j}, t\right)$ such that $(3.1)_{m+1}$ is satisfied. Suppose that $u_{m+1}(t)$ is already determined. Let \{\}$^{m+1}$ denote truncation of a power series so as to include terms in $t$ up to and including terms of degree $m+1$. For $z_{j} \in U_{i} \cap U_{j}$, let $z_{i}=g_{i j}\left(z_{j}, u^{m+1}(t)\right)$ and

$$
\psi_{i j}\left(z_{j}, t\right)=\left\{\varphi_{i}^{m}\left(z_{i}, t\right)-w_{i}\left(z_{i}\right)+\left[F_{i j}\left(z_{j}\right)+h_{i j}^{m}\left(z_{j}, t\right)\right]\left[w_{j}\left(z_{j}\right)-\varphi_{j}^{m}\left(z_{j}, t\right)\right]\right\}^{m+1} .
$$

Since (3.1) $m$ is satisfied, $\psi_{i j}\left(z_{j}, t\right)$ is in fact homogeneous of degree $m+1$.

Recall $\Phi$ of (2.2). Let $\Gamma\left(U_{i} \cap U_{j}, \Phi\right) t^{m+1}$ denote homogeneous polynomials of degree $m+1$ in $t$ with coefficients in $\Gamma\left(U_{i} \cap U_{j}, \Phi\right)$. Letting $y_{i}=\psi_{i j}\left(z_{j}, t\right)$, modulo Id $D$, gives an element of $\Gamma\left(U_{i} \cap U_{j}, \Phi\right) t^{m+1}$. We claim that $\left\{\psi_{i j}\right\}$, modulo $\operatorname{Id} D$, is in fact a cocycle. Let $z_{k} \in U_{i} \cap U_{j} \cap U_{k}$ and $z_{j}=f_{j k}\left(z_{k}\right)$. Then the cocycle condition may be written as

$$
\psi_{i k}\left(z_{k}, t\right) \equiv_{m+2} \psi_{i j}\left(z_{j}, t\right)+\tilde{F}_{i j}\left(z_{j}\right) \psi_{j k}\left(z_{k}, t\right) \bmod \left(\tilde{w}_{k}\left(z_{k}\right)\right) .
$$

Since the $\psi_{i j}$ are homogeneous of degree $m+1$, we may in fact verify (3.3) with the change of coordinates $z_{j} \equiv_{m+2} g_{j k}\left(z_{k}, u^{m+1}(t)\right)$. Then

$$
\begin{aligned}
z_{i} & \equiv{ }_{m+2} g_{i j}\left(z_{j}, u^{m+1}(t)\right) \equiv_{m+2} g_{i j}\left(g_{j k}\left(z_{k}, u^{m+1}(t)\right), u^{m+1}(t)\right) \\
& \equiv_{m+2} g_{i k}\left(z_{k}, u^{m+1}(t)\right) .
\end{aligned}
$$

We may also replace $\tilde{F}_{i j}$ and $\tilde{w}_{k}$ of (3.3) by $F_{i j}$ and $w_{k}$ respectively. Then, substituting from (3.2) and cancelling identical terms, we see that (3.3) is equivalent to

$$
\begin{aligned}
& -F_{i k}\left(z_{k}\right) \varphi_{k}^{m}\left(z_{k}, t\right)-h_{i k}^{m}\left(z_{k}, t\right) \varphi_{k}^{m}\left(z_{k}, t\right) \\
& \equiv{ }_{m+2} h_{i j}^{m}\left(z_{j}, t\right) w_{j}\left(z_{j}\right)-h_{i j}^{m}\left(z_{j}, t\right) \varphi_{j}^{m}\left(z_{j}, t\right) \\
& \quad-F_{i j}\left(z_{j}\right)\left[F_{j k}\left(z_{k}\right)+h_{j k}^{m}\left(z_{k}, t\right)\right] \varphi_{k}^{m}\left(z_{k}, t\right) \bmod \left(w_{k}\left(z_{k}\right)\right) .
\end{aligned}
$$

From $(3.1)_{m}$,

$$
F_{i k}\left(z_{k}\right)+h_{i k}^{m}\left(z_{k}, t\right) \equiv_{m+1}\left[F_{i j}\left(z_{j}\right)+h_{i j}^{m}\left(z_{j}, t\right)\right]\left[F_{j k}\left(z_{k}\right)+h_{j k}^{m}\left(z_{k}, t\right)\right] .
$$

Since $\varphi_{k}^{m}$ has terms in $t$ only of order at least one, (3.5) shows that

$$
\begin{aligned}
F_{i k}\left(z_{k}\right) \varphi_{k}^{m}\left(z_{k}, t\right)+ & h_{i k}^{m}\left(z_{k}, t\right) \varphi_{k}^{m}\left(z_{k}, t\right) \\
\equiv & { }_{m+2} F_{i j}\left(z_{j}\right)\left[F_{j k}\left(z_{k}\right)+h_{j k}^{m}\left(z_{k}, t\right)\right] \varphi_{k}^{m}\left(z_{k}, t\right) \\
& +h_{i j}^{m}\left(z_{j}, t\right)\left[F_{j k}\left(z_{k}\right)+h_{j k}^{m}\left(z_{k}, t\right)\right] \varphi_{k}^{m}\left(z_{k}, t\right) .
\end{aligned}
$$


Since $h_{i j}^{m}$ has terms in $t$ only of order at least one, (3.1) $)_{m}$ shows that

$$
\begin{aligned}
h_{i j}^{m}\left(z_{j}, t\right) w_{j}\left(z_{j}\right) & -h_{i j}^{m}\left(z_{j}, t\right) \varphi_{j}^{m}\left(z_{j}, t\right) \\
& \equiv{ }_{m+2} h_{i j}^{m}\left(z_{j}, t\right)\left[F_{j k}\left(z_{k}\right)+h_{j k}^{m}\left(z_{k}, t\right)\right]\left[w_{k}\left(z_{k}\right)-\varphi_{k}^{m}\left(z_{k}, t\right)\right] .
\end{aligned}
$$

Hence (3.4) and (3.3) hold.

Our numbering of obstructions is one higher than Kodaira's [12].

Let $H^{1}(D, \Phi) t^{m+1}$ denote the group of polynomials of degree $m+1$ in $t$ with coefficients in $H^{1}(D, \Phi)$.

Definition 3.1. Given $u^{m+1}(t)$ and $(3.1)_{m}, \operatorname{cls}\left[\psi_{i j}\right] \in H^{1}(D, \Phi) t^{m+1}$ is the $(m+$ 1)st obstruction to lifting $D$.

$m=0$ in Definition 3.1 agrees with our previous definition in Definition 2.3.

Proposition 3.2. Given $(3.1)_{m}$ and $u_{m+1}(t)$, there exist $\varphi_{i \mid m+1}\left(z_{i}, t\right)$ and $h_{i j \mid m+1}\left(z_{j}, t\right)$ satisfying $(3.1)_{m+1}$ if and only if the $(m+1)$ st obstruction vanishes.

Proof. The proof is the same as the proof of Proposition 2.4.

Proposition 3.3. Given $D$ and $u^{m+1}(t), \operatorname{cls}\left[\psi_{i j}\right] \in H^{1}(D, \Phi) t^{m+1}$ is independent of the choice of the $w_{i}\left(z_{i}\right)$ and of the $\varphi_{i}^{m}\left(z_{i}, t\right)$ satisfying $(3.1)_{m}$.

Proof. Different $w_{i}\left(z_{i}\right)$ may be regarded as additional $w_{i}\left(z_{i}\right)$ on a refinement of the original cover. This will not change any cohomology classes. So we always use the given $w_{i}\left(z_{i}\right)$.

Use primes ' to denote a different choice. We will first show, by induction on $p$, that for all $U_{i}$, there exist holomorphic $\theta_{i}^{m}$ on $U_{i}$,

$$
\theta_{i}^{m}\left(z_{i}, t\right)=\theta_{i \mid 1}\left(z_{i}, t\right)+\cdots+\theta_{i \mid p}\left(z_{i}, t\right)+\cdots+\theta_{i \mid m}\left(z_{i}, t\right)
$$

where $\theta_{i \mid p}\left(z_{i}, t\right)$ is homogeneous of degree $p$ in $t$, such that

$$
w_{i}\left(z_{i}\right)-\varphi_{i}^{\prime m}\left(z_{i}, t\right) \equiv_{m+1}\left(w_{i}\left(z_{i}\right)-\varphi_{i}^{m}\left(z_{i}, t\right)\right)\left(1+\theta_{i}^{m}\left(z_{i}, t\right)\right) .
$$

(3.6) holds trivially. So let us assume $(3.6)_{p}$ and prove $(3.6)_{p+1}, p+1<m$. $z_{i}=g_{i j}\left(z_{j}, u^{m+1}(t)\right) .(3.7)_{p+1}$ below says that the $(p+1)$ st obstruction vanishes.

$$
\begin{aligned}
\psi_{i j \mid p+1}\left(z_{j}\right) & =\left\{\varphi_{i}^{p}\left(z_{i}, t\right)-w_{i}\left(z_{i}\right)+\left[F_{i j}\left(z_{j}\right)+h_{i j}^{p}\left(z_{j}, t\right)\right]\left[w_{j}\left(z_{j}\right)-\varphi_{j}^{p}\left(z_{j}, t\right)\right]\right\}^{p+1} \\
& =\left\{-\varphi_{i \mid p+1}\left(z_{i}, t\right)+F_{i j}\left(z_{j}\right) \varphi_{j \mid p+1}\left(z_{j}, t\right)-h_{i j \mid p+1}\left(z_{j}, t\right) w_{j}\left(z_{j}\right)\right\}^{p+1}
\end{aligned}
$$

$1 /\left(1+\theta_{j}^{m}\left(z_{j}, t\right)\right)=\Sigma(-1)^{\nu}\left[\theta_{j}^{m}\left(z_{j}, t\right)\right]^{\nu}, \nu \geqslant 0$. Since $\psi_{i j \mid p+1}$ is in fact homogeneous of degree $p+1$ in $t$,

$$
\begin{aligned}
\psi_{i j \mid p+1}\left(z_{j}\right)=\left\{\left[\varphi_{i}^{p}\left(z_{i}, t\right)-w_{i}\left(z_{i}\right)\right]\left[1+\theta_{i}^{p}\left(z_{i}, t\right)\right]\right. \\
\left.+\left[F_{i j}\left(z_{j}\right)+h_{i j}^{p}\left(z_{j}, t\right)\right]\left[1+\theta_{i}^{p}\left(z_{i}, t\right)\right]\left[w_{j}\left(z_{j}\right)-\varphi_{j}^{p}\left(z_{j}, t\right)\right]\right\}^{p+1} \\
=\left\{\left[\varphi_{i}^{p}\left(z_{i}, t\right)-w_{i}\left(z_{i}\right)\right]\left[1+\theta_{i}^{p}\left(z_{i}, t\right)\right]\right. \\
+\left[F_{i j}\left(z_{j}\right)+h_{i j}^{p}\left(z_{j}, t\right)\right]\left[1+\theta_{i}^{p}\left(z_{i}, t\right)\right] /\left[1+\theta_{j}^{p}\left(z_{j}, t\right)\right] \\
\left.\cdot\left[w_{j}\left(z_{j}\right)-\varphi_{j}^{p}\left(z_{j}, t\right)\right]\left[1+\theta_{j}^{p}\left(z_{j}, t\right)\right]\right\}^{p+1} .
\end{aligned}
$$


From (3.6),

$$
\begin{array}{r}
F_{i j}\left(z_{j}\right)+h_{i j}^{\prime p}\left(z_{j}, t\right)=\left\{\left[F_{i j}\left(z_{j}\right)+h_{i j}^{p}\left(z_{j}, t\right)\right] \cdot\left[1+\theta_{i}^{p}\left(z_{i}, t\right)\right] /\left[1+\theta_{j}^{p}\left(z_{j}, t\right)\right]\right\}^{p} \\
\begin{array}{r}
\psi_{i j \mid p+1}\left(z_{j}\right)-\psi_{i j \mid p+1}^{\prime}\left(z_{j}\right) \\
\equiv\left\{\varphi_{i \mid 1}\left(z_{i}, t\right) \theta_{i \mid p}\left(z_{i}, t\right)+\cdots+\varphi_{i \mid p}\left(z_{i}, t\right) \theta_{i \mid 1}\left(z_{i}, t\right)\right. \\
\left.\quad-F_{i j}\left(z_{j}\right)\left[\varphi_{j \mid 1}\left(z_{j}, t\right) \theta_{j \mid p}\left(z_{j}, t\right)+\cdots+\varphi_{j \mid p}\left(z_{j}, t\right) \theta_{j \mid 1}\left(z_{j}, t\right)\right]\right\}^{p+1} \\
\bmod \left(w_{j}\left(z_{j}\right)\right) .
\end{array}
\end{array}
$$

Since $\Gamma(D, \Phi)=0, \psi_{i j \mid p+1}^{\prime}\left(z_{j}\right)$ is a coboundary in a unique way, modulo $\left(w_{j}\left(z_{j}\right)\right)$. From $(3.7)_{p+1}$ and (3.8) $)_{p+1}$,

$$
\begin{aligned}
& \psi_{i j \mid p+1}^{\prime}\left(z_{j}, t\right) \equiv\left\{-\varphi_{i \mid p+1}^{\prime}\left(z_{i}, t\right)+F_{i j}\left(z_{j}\right) \varphi_{j \mid p+1}^{\prime}\left(z_{j}\right)\right\}^{p+1} \\
& \equiv\left\{-\varphi_{i \mid p+1}\left(z_{i}, t\right)-\varphi_{i \mid 1}\left(z_{i}, t\right) \theta_{i \mid p}\left(z_{i}, t\right)-\cdots-\varphi_{i \mid p}\left(z_{i}, t\right) \theta_{i \mid 1}\left(z_{i}, t\right)\right. \\
&+F_{i j}\left(z_{j}\right)\left[\varphi_{j \mid p+1}\left(z_{j}, t\right)+\varphi_{j \mid 1}\left(z_{j}, t\right) \theta_{j \mid p}\left(z_{j}, t\right)\right. \\
&\left.\left.+\cdots+\varphi_{j \mid p}\left(z_{j}, t\right) \theta_{j \mid 1}\left(z_{j}, t\right)\right]\right\}^{p+1} \\
& \bmod \left(w_{j}\left(z_{j}\right)\right) .
\end{aligned}
$$

Then for each $i$, there is a $\theta_{i \mid p+1}\left(z_{i}, t\right)$, homogeneous in $t$ of degree $p+1$, such that

$$
\begin{aligned}
\varphi_{i \mid p+1}^{\prime}\left(z_{i}, t\right)= & \varphi_{i \mid p+1}(z, t)+\varphi_{i \mid 1}\left(z_{i}, t\right) \theta_{i \mid p}\left(z_{i}, t\right) \\
& +\cdots+\varphi_{i \mid p}\left(z_{i}, t\right) \theta_{i \mid 1}\left(z_{i}, t\right)+w_{i}\left(z_{i}\right) \theta_{i \mid p+1}\left(z_{i}, t\right) .
\end{aligned}
$$

This is precisely what is needed to derive $(3.6)_{p+1}$ from $(3.6)_{p}$. So (3.6) $)_{m}$ is true. Also $(3.8)_{m+1}$ is true. Thus, $\operatorname{cls}\left[\psi_{i j}\right]=\operatorname{cls}\left[\psi_{i j}^{\prime}\right]$, which completes the proof of the proposition.

Since Proposition 2.2 allows the possibility of nonsmooth parameter spaces for weak liftings, we would like to say something directly in this case. For $\boldsymbol{R}$ a subvariety of $Q$, let $C(R, 0)=C_{3}(R, 0)$ be the Whitney tangent cone [29]. Recall $\delta$ of Definition 2.3.

Proposition 3.4. Let $\omega:$ T $\rightarrow Q$ be a 1-convex map which gives a deformation of the two-dimensional manifold $M=\omega^{-1}(0)$. Let $R$ be a subvariety of $Q$ to which $a$ cycle $D>0$ on $A$ weakly lifts locally. Let $\nu \in C(R, 0)$. Then $\delta(\nu)=0$.

Proof. By [29, Lemma 11.2, p. 517] there is an irreducible one-dimensional subvariety $S$ of $R$ near 0 such that $\nu \in C(S, 0)$. Let $\pi: T \rightarrow S$ be a resolution of $S$. Let $t$ be a local parameter for $T$ near $0=\pi^{-1}(0)$. Choose coordinates $q=$ $\left(q_{1}, \ldots, q_{n}\right)$ for $Q$ so that $\nu$ is in the direction of the $q_{1}$-axis. Then, since $S$ is irreducible and of dimension 1 , there is an integer $p>1$ so that $\pi(t)$ $\equiv_{p+1}\left(c t^{p}, 0, \ldots, 0\right)$ with $c \in \mathbf{C}-\{0\}$. By multiplying $t$ by $c^{1 / p}$, we may assume that $c=1$. Let $\tau: \Re \rightarrow T$ be the pull-back of $\omega$ via $\pi$. Then on $\Re$, modulo $t^{p+1}$, the change of coordinates is given by $z_{1}=g_{i j}\left(z_{j}, t^{p}\right)$. In (2.3), $\varphi_{i \mid m}$ and $h_{i j \mid m}$ may be 
chosen to be 0 for $1 \leqslant m \leqslant p-1$. Let $\left\{\lambda_{i j}\right\}$ be the cycle in $H^{1}(N(\mathfrak{U}), \Theta)$ corresponding to $\nu$. (2.5) is replaced by

$$
\left(t^{p} \cdot \lambda_{i j} \tilde{w}_{i}\right)\left(z_{i}\right)-\varphi_{i \mid p}\left(z_{i}, t\right)=-\tilde{F}_{i j}\left(z_{j}\right) \varphi_{j \mid p}\left(z_{j}, t\right)+h_{i j \mid p}\left(z_{j}, t\right) \tilde{w}_{j}\left(z_{j}\right) ; \quad z_{i}=f_{i}\left(z_{j}\right) .
$$

$D$ lifts to $T$ locally. By Proposition 3.3, the $p$ th obstruction to lifting $D$ is well defined and vanishes. So the above replacement for (2.5) can be solved. Hence $\delta(\nu)=0$.

TheORem 3.5. Let $D$ be a cycle on the exceptional set of a strictly pseudoconvex manifold $M$. Then

(i) $\beta_{*}: H^{1}(M, \Theta) \rightarrow H^{1}(D, \Phi)$ is onto if and only if $H^{1}(D, \mathcal{T})=0$.

Let $\omega: \mathfrak{T} \rightarrow Q$ be a deformation of $M$ with $Q$ smooth. Suppose that $\delta:{ }_{Q} T_{0} \rightarrow$ $H^{1}(D, \Phi)$ is onto. Then

(ii) There is a submanifold $S$ of $Q$ with ${ }_{s} T_{0}=\operatorname{ker} \delta$ and a subspace $\mathscr{D}$ of $\mathfrak{R}$ such that $\lambda=\omega \mid \mathscr{D}: \mathscr{D} \rightarrow S$ is a lifting of $D$ locally.

(iii) $S$ is the maximal subspace of $Q$ to which $D$ locally lifts weakly.

Suppose that additionally $\rho_{0}: Q^{T_{0}} \rightarrow H^{1}(M, \Theta)$ is onto. Then

(iv) $\lambda: \mathscr{D} \rightarrow S$ is a complete deformation of $D$ as an abstract (nonembedded) analytic space.

(v) Off a proper subvariety of $S, D_{s}=\lambda^{-1}(s)$ consists of finitely many disjoint (reduced, nonsingular) Riemann surfaces.

Proof. (i) follows immediately from (2.8) and (2.9).

We shall first construct $S$ of (ii) formally and then prove convergence. $S$ will be given as the biholomorphic image of a manifold $T$ under a map $u: T \rightarrow Q$, $u(0)=0$. Let $n$ be the dimension of ker $\delta$. Let $t=\left(t_{1}, \ldots, t_{n}\right)$ be coordinates near 0 in $T$. Let $u_{1}(t)$ be any linear map whose image is ker $\delta$. We may choose coordinates $q=\left(q_{1}, \ldots, q_{n}, q_{n+1}, \ldots, q_{n+d}\right), d=\operatorname{dim} H^{1}(D, \Phi)$ so that $u_{1}\left(t_{1}, \ldots, t_{n}\right)=\left(t_{1}, \ldots, t_{n}, 0, \ldots, 0\right)$. Identify $Q$ with its tangent space at 0 . Then $\delta:\left\{\left(0, \ldots, 0, q_{n+1}, \ldots, q_{n+d}\right)\right\} \rightarrow H^{1}(D, \Phi)$ is an isomorphism. Proposition 2.4 says that $(3.1)_{1}$ can be satisfied. We now continue by induction on $m$, assuming that $u^{m}(t), \varphi_{i}^{m}\left(z_{i}, t\right)$ and $h_{i j}^{m}\left(z_{j}, t\right)$ have been found for all $U_{i}$ and $U_{i} \cap U_{j}$ and that (3.1) $)_{m}$ holds.

Let $\operatorname{cls}\left[\psi_{0}\right] \in H^{1}(D, \Phi) t^{m+1}$ be the $(m+1)$ st obstruction to lifting $D$ to $T$ for $u_{m+1}(t)=0$. For each coefficient $c$ in $\operatorname{cls}\left[\psi_{0}\right]$, there is a unique $q=$ $\left(0, \ldots, 0, q_{n+1}, \ldots, q_{n+d}\right) \in Q$ such that $\delta(q)=c$. Let $u_{m+1}(t)$ be redefined so that the corresponding coefficient is $-q$. Since $\varphi_{i}^{m}\left(z_{i}, t\right)$ has terms in $t$ only of order at least one, (3.2) shows that for the new $u_{m+1}(t), \operatorname{cls}\left[\psi_{i j}\right]=0$. Hence $(3.1)_{m+1}$ can be satisfied.

We shall now prove convergence. We will be modifying Kodaira's proof for nonsingular $D$ [12, Theorem 1, pp. 150-158]. We first reduce to the case where the $A_{i}$, the irreducible components of the exceptional set $A$ of $D$, are nonsingular and have normal crossings. So, if $x \in A$ is any point where an $A_{i}$ is singular or there are not normal crossings, take any section of $\mathscr{T}$ above $Q$ and through $x$. Perform a monoidal transformation $\pi: \mathfrak{N}^{\prime} \rightarrow \mathfrak{K}$ along the image of the section. This induces 
a quadratic transformation on each $M_{q}$ and in particular on $M=M_{0}$ at $x$. $\omega^{\prime}=\omega \circ \pi: \Re^{\prime} \rightarrow Q$ is a deformation of $M^{\prime}=\left(\omega^{\prime}\right)^{-1}(0)$. Let $D^{\prime}$ be the total transform on $M^{\prime}$ of $D$ on $M$. Then by [18, Proposition 4.7, p. 26] $\delta^{\prime}: Q^{T_{0}} \rightarrow$ $H^{1}\left(D^{\prime}, \Phi^{\prime}\right)$ is onto. Suppose that we have proved (ii) for $D^{\prime}$. Look at $\mathscr{D}^{\prime}$ near $\pi^{-1}(x)=A_{0}^{\prime} . \omega^{\prime}$ is a trivial deformation near $A_{0}^{\prime}$. There is a small neighborhood $U^{\prime}$ of $A_{0}^{\prime}$ in $M^{\prime}$ such that $H^{1}\left(U^{\prime}, \theta\right)=0$. Let $V^{\prime}$ be a Cartesian product neighborhood of $U^{\prime}$ in $\left(\omega^{\prime}\right)^{-1}(S)$. We may take $H^{1}\left(V^{\prime}, \theta\right)=0$. Since $\mathscr{D}^{\prime}$ is a topologically trivial divisor in $V^{\prime}$, it is also analytically trivial in $V^{\prime}$ with a single defining equation $w^{\prime}$. Let $\pi: V^{\prime} \rightarrow V$ be the blow down of $V^{\prime}$ under $\pi$. Then $w^{\prime}=\pi^{*}(w)$ for a holomorphic function on $V$. Also, $D$ is the divisor of $w$ on $M$ near $x$. Thus, we can find equations which define $\mathscr{D}^{\prime}$ on $\left(\omega^{\prime}\right)^{-1}(S)$ which blow down to define $\mathscr{D}$ on $\omega^{-1}(S)$. So it suffices to prove (ii) for $D^{\prime}$. After finitely many quadratic transformations, we may assume nonsingular $A_{i}$ and normal crossings.

We first construct a particular special cover $\mathfrak{U}=\left\{U_{i}\right\}, 0 \leqslant i<s$, of a neighborhood of $A$. Each $U_{i}$ will be privileged for $\Phi$ and $U_{i} \cap U_{j}$ will also behave well. Choose a finite set $B=\left\{b_{i}\right\}, 1<i<s$, of points in $A$ such that $B$ includes all singular points of $A$ and at least one point in each $A_{i}$. Choose a small neighborhood $U_{i}$ of each $b_{i}$ such that $U_{i}$ is a polydisc in local coordinates $z_{i}=\left(x_{i}, y_{i}\right)$ and either $A \cap U_{i}=\left\{x_{i} y_{i}=0\right\}$ or $A \cap U_{i}=\left\{y_{i}=0\right\}$. Let $V_{i}$ be a smaller polydisc with $b_{i} \in V_{i}$ and $V_{i} \subset \subset U_{i}, 1 \leqslant i<s$. We choose $U_{0}$ as a neighborhood in $M$ of $R=A-\cup \bar{V}_{i}, 1<i \leqslant s, \bar{R} \cap B=\varnothing$, which is isomorphic to the Cartesian product of $R$ and a disc as follows. Perform quadratic transformations at sufficiently many $b_{i}$, increasing the number of $b_{i}$ if necessary, so that the proper transforms $A_{j}^{\prime}$ of the $A_{j}$ satisfy $A_{j}^{\prime} \cap A_{k}^{\prime}=\varnothing, j \neq k$, and have very large self-intersections, i.e. so that in [6, Corollary, p. 363], all obstructions to extending isomorphisms near the $A_{j}^{\prime}$ vanish. Then each $A_{j}^{\prime}$ has a neighborhood isomorphic to a neighborhood of the zero section of its normal bundle $N_{j}$. We may regard $A_{j}-B$ as an open Riemann surface and so Stein [10, Theorem IX.C.10, p. 270]. Moreover, since $H^{1}\left(A_{j}-B, \theta\right)=0$ and $H^{2}\left(A_{j}-B ; Z\right)=0, N_{j}$ is the trivial bundle over $A_{j}-B$. So we may choose $U_{0}$ as a Cartesian product of $R$ and a disc. Let $x_{0}$ denote a typical point in $R$ and $y_{0}$ a coordinate for the disc. $z_{0} \in U_{0}$ is given by $z_{0}=\left(x_{0}, y_{0}\right)$. So that we may add small quantities to $x_{0}$, immerse $R$ in $\mathbf{C}$ [9]. We then have a coordinate cover for $R$ in which each change of coordinates is the identity.

Following Kodaira, we let

$$
A(t)=\frac{a}{16 b} \sum \frac{1}{m^{2}} b^{m}\left(t_{1}+\cdots+t_{n}\right)^{m}, \quad 1<m<\infty,
$$

where $a$ and $b$ are positive real constants to be chosen later. If $\xi(z, u)=$ $\sum \xi_{l m \ldots n}(z) u_{1}^{l} u_{2}^{m} \ldots u_{q}^{n}$ is a power series in $u_{1}, u_{2}, \ldots, u_{q}$ whose coefficients carry a norm $\left\|\xi_{l m} \ldots n(z)\right\|$ and if $a(u)=\sum a_{l m} \ldots n u_{1}^{l} u_{2}^{m} \ldots u_{q}^{n}$ has $a_{l m \ldots n}>0$, we shall write $\xi(z, u) \ll a(u)$ to mean $\left\|\xi_{l m \ldots n}(z)\right\|<a_{l m \ldots n}$ for all $l, m, \ldots, n$. Note that

$$
A(t)^{\nu} \ll(a / b)^{\nu-1} A(t) \text { for } \nu \geqslant 2 \text {. }
$$


We wish to solve (3.1) $m$ for all $m$, as before, and show that for all $m, i, i j$, we may choose $u^{m}(t), \varphi_{i}^{m}\left(z_{i}, t\right)$ and $h_{i j}^{m}\left(z_{j}, t\right)$ such that

$$
u^{m}(t) \ll A(t), \quad \varphi_{i}^{m}\left(z_{i}, t\right) \ll A(t), \quad h_{i j}^{m}\left(z_{j}, t\right) \ll A(t) .
$$

In $(3.10)_{m}$, the norm for $u_{m}(t)$ is the maximum over the absolute value of the components. For $\varphi_{i \mid m}\left(z_{i}, t\right)$ and $h_{i j \mid m}\left(z_{j}, t\right)$, it is the supremum over $z_{i} \in U_{i}$ and $z_{j} \in U_{i} \cap U_{j}$ respectively. Working initially on a larger cover than $\mathfrak{U}$ and then shrinking, we may assume that $\varphi_{i \mid 1}\left(z_{i}, t\right)$ and $h_{i j \mid 1}\left(z_{j}, t\right)$ have bounded coefficients. Then for sufficiently large $a,(3.10)_{1}$ holds. We now assume by induction that $(3.10)_{m}$ holds.

We use the following norm || on sections of $\Phi$. Different local coordinates will give equivalent norms. Let $V_{k}^{\prime}$ be a local polydisc coordinate patch with coordinates $z_{k}=\left(x_{k}, y_{k}\right)$ and suppose Id $D=\left(y_{k}^{d}\right) \cdot \Phi$ is the sheaf of germs of sections of the trivial line bundle on $V_{k}^{\prime}$. Let $V_{k} \subset \subset V_{k}^{\prime}$ be a smaller polydisc. Any $\varphi \in$ $\Gamma\left(V_{k}, \Phi\right)$ corresponds to a $\varphi_{1} \in \Gamma\left(V_{k}, \mathcal{\theta} /\left(y_{k}^{d}\right)\right)$. Let $\varphi_{2} \in \Gamma\left(V_{k}, \theta\right)$ be an ambient representative for $\varphi_{1}$. Let ${ }^{(\nu)}$ denote the $\nu$ th partial derivative with respect to $y_{k}$. Let

$$
|\varphi|_{V_{k}}=\max \sup \left|\varphi_{2}^{(\nu)}\left(z_{k}\right)\right|, \quad z_{k}=\left(x_{k}, 0\right) \in V_{k}, \quad 0<\nu<d-1 .
$$

$|\varphi|_{V_{k}}$ is independent of the choice of $\varphi_{2}$. The other case for Id $D$ is Id $D=\left(x_{k}^{e} y_{k}^{d}\right)$. Let $V_{1}=V_{k}-\{x=0\}$ and $V_{2}=V_{k}-\{y=0\}$. Then let $|\varphi|_{V_{k}}=$ $\max \left(|\varphi|_{V_{1}},|\varphi|_{V_{2}}\right)$. For an arbitrary set $U$, cover $\bar{U}$ by finitely many coordinate patches $V_{k}$. Then $|\varphi|_{U}=\max |\varphi|_{V_{k} \cap U}$. Different covers give equivalent norms.

We first let $u_{m+1}(t)=0$ and estimate $\left|\psi_{0}\right|$ for $\psi_{0}$ given by (3.2) as a cocycle in $C^{1}(N(\mathfrak{U}), \Phi)$. Let $\psi_{i j 0}\left(z_{j}\right)$ denote $\psi_{0}$ on $U_{i} \cap U_{j}$. From the Cartesian product structure, Cauchy's integral formula for derivatives, and the maximum principle, there is a constant $c_{1}$ such that if $\Psi_{i j}\left(z_{j}\right) \in \Gamma\left(U_{i} \cap U_{j}, \theta\right) t^{m+1}$ restricts to $\psi_{i j 0}\left(z_{j}\right)$ on $D$,

$$
\left|\psi_{i j 0}\left(z_{j}\right)\right|<c_{1}\left\|\Psi_{i j}\left(z_{j}\right)\right\|, \quad z_{j} \in U_{i} \cap U_{j} .
$$

This $c_{1}$ and future $c_{k}$ will be independent of $m$. Let \{\}$_{m+1}$ denote the terms of order $m+1$ in $t$. Then we may take

$$
\begin{aligned}
\Psi_{i j}\left(z_{j}\right) & =\left\{\varphi_{i}^{m}\left(z_{i}, t\right)-w_{i}\left(z_{i}\right)-h_{i j}^{m}\left(z_{j}, t\right) \varphi_{j}^{m}\left(z_{j}, t\right)\right\}_{m+1}, \\
z_{i} & =g_{i j}\left(z_{j}, u^{m}(t)\right) .
\end{aligned}
$$

By (3.10) $m$ and (3.9),

$$
h_{i j}^{m}\left(z_{j}, t\right) \varphi_{j}^{m}\left(z_{j}, t\right) \ll a b^{-1} A(t), \quad z_{j} \in U_{i} \cap U_{j} .
$$

By starting with a larger cover and then shrinking, we may assume that $\omega$ is a trivial deformation on each $U_{i}$. Recall our notation $z_{i}=g_{i j}\left(z_{j}, q\right), q \in Q$, for the transition maps; $f_{i j}\left(z_{j}\right)=g_{i j}\left(z_{j}, 0\right)$. Recall that $r=n+d$ is the dimension of $Q$. By shrinking the cover, we may also assume that there are constants $c_{2}$ and $c_{3}$ such that, with $\eta=\left(\eta_{1}, \eta_{2}\right)$

$$
\begin{aligned}
g_{i j}\left(z_{j}, q\right)-f_{i j}\left(z_{j}\right) \ll \sum c_{2}^{\nu}\left(q_{1}+\cdots+q_{\nu}\right)^{\nu}, & 1<\nu<\infty, z_{j} \in U_{i} \cap U_{j}, \\
w_{i}\left(z_{i}+\eta\right)-w_{i}\left(z_{i}\right) \ll \sum c_{3}^{\tau}\left(\eta_{1}+\eta_{2}\right)^{\tau}, & 1<\tau<\infty, z_{i} \in U_{i} .
\end{aligned}
$$


Since $q=u^{m}(t) \ll A(t)$,

$$
g_{i j}\left(z_{j}, u^{m}(t)\right)-f_{i j}\left(z_{j}\right) \ll \frac{c_{2} r A(t)}{1-c_{2} r(a / b)} .
$$

By choosing $b$ to be sufficiently large with respect to $a$ so that $1-c_{2} r a / b>\frac{1}{2}$ we may assume that

$$
g_{i j}\left(z_{j}, u^{m}(t)\right)-f_{i j}\left(z_{j}\right) \ll 2 c_{2} r A(t) .
$$

Let $\eta=g_{i j}\left(z_{j}, u^{m}(t)\right)-f_{i j}\left(z_{j}\right)$. From (3.15), $w_{i}\left(g_{i j}\left(z_{j}, u^{m}(t)\right)\right)-w_{i}\left(f_{i j}\left(z_{j}\right)\right) \ll$ $\sum c_{3}^{\tau}\left(\eta_{1}+n_{2}\right), 1 \leqslant \tau<\infty, z_{j} \in U_{i} \cap U_{j}$. Since $q$ contains no terms in $t$ of order $m+1$, in estimating $\left\{w_{i}\left(z_{i}\right)\right\}_{m+1}$ from (3.14) and (3.15), for $\tau=1$ we may start the summation in $\nu$ from $\nu=2$. Then, also using (3.16)

$$
\left\{w_{i}\left(g_{i j}\left(z_{j}, u^{m}(t)\right)\right)\right\}_{m+1} \ll 4 c_{2}^{2} c_{3} r a b^{-1} A(t)+\frac{16 c_{2}^{2} c_{3}^{2} r^{2}(a / b) A(t)}{1-4 c_{2} c_{3} r(a / b)} .
$$

With $1-4 c_{2} c_{3} r(a / b)>\frac{1}{2}$, there is a constant $c_{4}$ with

$$
\left\{w_{i}\left(g_{i j}\left(z_{j}, u^{m}(t)\right)\right)\right\}_{n+1} \ll c_{4} a b^{-1} A(t) .
$$

Finally, we must estimate $\varphi_{i}^{m}\left(z_{i}, t\right)$ from (3.12). Let $U_{i}^{\prime} \subset \subset U_{i}$ and $\delta>0$ be chosen so that, for $z_{i} \in U_{i}^{\prime}$, the polydisc $\Delta$ centered at $z_{i}$ with polyradius $\delta$ satisfies $\Delta \subset U_{i}$, and so that $\left\{U_{i}^{\prime}\right\}$ is a cover for $A$. Let $\eta=\left(\eta_{1}, \eta_{2}\right)$. Then for $z_{i} \in U_{i}^{\prime}$, $\varphi_{i}^{m}\left(z_{i}+\eta, t\right) \ll A(t)\left(1-\eta_{1} / \delta\right)^{-1}\left(1-\eta_{2} / \delta\right)^{-1}$. Let $\eta=g_{i j}\left(z_{j}, u^{m}(t)\right)-f_{i j}\left(z_{j}\right)$. Then calculating as in [12, p. 156], there is a constant $c_{5}$ such that, for sufficiently large $b$ with respect to $a$,

$$
\left\{\varphi_{i}^{m}\left(g_{i j}\left(z_{j}, u^{m}(t)\right), t\right)\right\}_{m+1} \ll c_{5} a b^{-1} A(t), \quad z_{j} \in U_{i}^{\prime} \cap U_{j} .
$$

From (3.11), (3.12), (3.13), (3.17) and (3.18), $\psi_{i j 0}\left(z_{j}\right) \ll c_{1}\left(1+c_{4}+c_{5}\right) a b^{-1} A(t)$, $z_{j} \in U_{i}^{\prime} \cap U_{j}$. From the cocycle condition on $\psi_{i j 0}$, there is a constant $c_{6}$ so that, as in [12, pp. 156-157],

$$
\psi_{i j 0}\left(z_{j}\right) \ll c_{1}\left(1+c_{4}+c_{5}\right) c_{6} a b^{-1} A(t), \quad z_{j} \in U_{i} \cap U_{j} .
$$

We may compute $H^{1}(D, \Phi)$ using bounded cochains from $N(\mathfrak{U})$; see [13, §6, pp. 499-500]. Give $H^{1}(D, \Phi)$ the quotient norm. Let $c_{7}=c_{1}\left(1+c_{4}+c_{5}\right) c_{6}$. Then for $\operatorname{cls}\left[\psi_{0}\right] \in H^{1}(D, \Phi) t^{m+1}, \operatorname{cls}\left[\psi_{0}\right] \ll c_{7} a b^{-1} A(t)$. There is a constant $c_{8}$ such that

$$
u_{m+1}(t) \ll c_{7} c_{8} a b^{-1} A(t) .
$$

Now let $\psi_{i j}$ be computed with $u^{m+1}(t)=u^{m}(t)+u_{m+1}(t)$. Then there is a constant $c_{9}$ such that

$$
\psi_{i j}\left(z_{j}\right) \ll\left(c_{7}+c_{7} c_{8} c_{9}\right) a b^{-1} A(t) .
$$

$\operatorname{cls}\left(\psi_{i j}\right)=0$ in $H^{1}(D, \Phi)$. Then, as in [12, Lemma, p. 157], there is a constant $c_{10}$ so that we may choose $\varphi_{i \mid m+1}^{\prime}\left(z_{i}, t\right) \in \Gamma\left(U_{i}, \Phi\right) t^{m+1}$ such that

$$
\psi_{i j}\left(z_{j}\right)=-F_{i j}\left(z_{j}\right) \varphi_{j \mid m+1}^{\prime}\left(z_{j}, t\right)+\varphi_{i \mid m+1}^{\prime}\left(z_{i}, t\right), \quad z_{i}=f_{i j}\left(z_{j}\right),
$$

and $\varphi_{i \mid m+1}^{\prime} \ll c_{10}\left(c_{7}+c_{7} c_{8} c_{9}\right) a b^{-1} A(t)$. From the Cartesian product choices for our cover $\mathfrak{U}$, there is a constant $c_{11}$ such that each $\varphi_{i \mid m+1}^{\prime}\left(z_{i}, t\right)$ has an ambient representative $\varphi_{i \mid m+1}\left(z_{i}, t\right) \in \Gamma\left(U_{i}, \theta\right) t^{m+1}$ with

$$
\varphi_{i \mid m+1}\left(z_{i}, t\right) \ll c_{11} c_{10}\left(c_{7}+c_{7} c_{8} c_{9}\right) a b^{-1} A(t) .
$$


$(3.1)_{m+1}$ is now satisfied modulo $\left(w_{j}\left(z_{j}\right)\right)$. Let $z_{i}=g_{i j}\left(z_{j}, u^{m+1}(t)\right)$ and

$$
\begin{aligned}
h_{i j \mid m+1}\left(z_{j}, t\right)=\frac{1}{w_{j}\left(z_{j}\right)}\{ & w_{i}\left(z_{i}\right)-\varphi_{i}^{m+1}\left(z_{i}, t\right) \\
& \left.-\left[F_{i j}\left(z_{j}\right)+h_{i j}^{m}\left(z_{j}, t\right)\right]\left[w_{j}\left(z_{j}\right)-\varphi_{j}^{m+1}\left(z_{j}, t\right)\right]\right\}^{m+1} .
\end{aligned}
$$

Since $(3.1)_{m+1}$ is satisfied, $h_{i j \mid m+1}\left(z_{j}, t\right)$ is homogeneous of degree $m+1$ as desired. Since $\varphi_{i \mid m+1}\left(z_{i}, t\right)$ is homogeneous of degree $m+1$ in $t$,

$$
\left\{\varphi_{i \mid m+1}\left(g_{i j}\left(z_{j}, u^{m+1}(t)\right), t\right)\right\}^{m+1}=\varphi_{i \mid m+1}\left(f_{i j}\left(z_{j}\right), t\right) \text { for } z_{i}=f_{i j}\left(z_{j}\right) \in U_{i} \cap U_{j} \text {. }
$$

Then by (3.20) and (3.21), there is a constant $c_{12}$ such that the numerator of (3.22) is dominated by $c_{12} a b^{-1} A(t)$. By the construction of $\mathfrak{U}$ and the maximum principle, functions on $U_{i} \cap U_{j}$ may be estimated by their values away from $A$. Then there is a constant $c_{13}$ such that

$$
h_{i j \mid m+1}\left(z_{j}, t\right) \ll c_{13} c_{12} a b^{-1} A(t) .
$$

We now complete the induction step and the proof of (ii) by choosing $a b^{-1}$ sufficiently small so that the coefficients of $A(t)$ in (3.19), (3.21) and (3.23) are all less than 1 .

By [23, Satz 1, p. 547], we may assume that $\omega$ is 1-convex.

Let $R$ be the maximum subspace of $Q$, given by Proposition 2.2, to which $D$ weakly lifts locally. We need that $R \subset S$. If not, there is a one-dimensional disc $T$ and a holomorphic map $u: T \rightarrow Q, u(0)=0, u(T) \nsubseteq S$, and such that $D$ lifts locally to $T$ on the deformation of $M$ induced from $\omega$ by $u$. Choose local coordinates on $Q$ so that $S$ is a linear subspace of $Q \cdot u(t)=u_{1}(t)+\cdots+u_{p}(t)$ $+\cdots$. Since $u(T) \notin S$, there is a least $m \geqslant 0$ such that the image of $u_{m+1}(t)$ is not in $S$. Since $D$ lifts to $S$, the $(m+1)$ st obstruction $\left[\psi_{0}\right]$, well defined by Proposition 3.4, to lifting $D$ to $T$ with $u=u^{m}(t)$ vanishes. Since $D$ lifts to $T$, the $(m+1)$ st obstruction $[\psi]$ to lifting $D$ to $T$ with $u=u^{m+1}(t)$ also vanishes. But, as in the proof of (ii), $\left[\psi-\psi_{0}\right]=\delta\left(u_{m+1}(t)\right)$. Since ${ }_{s} T_{0}=\operatorname{ker} \delta$, the image of $u_{m+1}(t)$ is contained in $S$. This contradicts our minimal choice of $m$. Hence $R \subset S$ as needed to prove (iii).

By Proposition 2.10, $S$ is an infinitesimally complete deformation of $D$. Let $S_{1}$ be a submanifold of $S$ such that $s_{1} T_{0}$ is isomorphic to the infinitesimal deformations of $D$. Then the map from $S_{1}$ to the parameter space for the versal deformation of $D$ [8], [3] is an isomorphism since $S_{1}$ is smooth. Hence $S_{1}$ and also $S$ is a complete deformation of $D$.

By the openness of completeness [8, Hauptsatz, p. 140], $\lambda$ is a complete deformation of $D_{s}$ for any $s \in S$ near 0 . Let $W$ be the reduction of $\mathscr{D}$. Let $\lambda_{1}: W \rightarrow S$ be the induced deformation from $\lambda$. Then, as in the proofs of [18, Theorem 2.1, p. 3 and Theorem 2.2, p. 4], off a proper subvariety $S_{1}$ of $S, W_{s}$ is reduced and $\lambda_{1}$ gives an equisingular deformation of the singular points of $W_{s}$. We will first show that in fact for $s \notin S_{1}, W_{s}$ is nonsingular. Suppose that $x$ is a singular point of $W_{s}, s \notin S_{1}$. Choose local coordinates $z$ with $x=0$. Let $f(z)$ generate $\operatorname{Id}\left(D_{s}\right)$ near 0 . Let

$$
f(z)=f_{1}^{q_{1}}(z) \ldots f_{k}^{q_{k}}(z)
$$


be a factorization of $f$ into prime factors; $f_{i}$ and $f_{j}$ are not associates for $i \neq j$. Since (3.24) may be obtained by decomposing $D_{s}$ into irreducible components, since $\lambda_{1}$ is an equisingular deformation, and since $\mathscr{D}$ differs from $W$ only in that the local defining equations for $\mathscr{D}$ may have multiple factors, $\mathscr{D}$ has a local defining equation

$$
g(z, s)=g_{1}^{q_{1}}(z, s) \ldots g_{k}^{q_{k}}(z, s)
$$

with $g_{i}(z, 0)=f_{i}(z)$.

Consider the following nonzero infinitesimal deformation $\lambda_{2}$ of $D_{s}$, with a parameter space $\varepsilon / \varepsilon^{2}, \varepsilon$ one-dimensional. In $z$-coordinates, $\lambda_{2}$ is given by

$$
h(z, \varepsilon)=f_{1}^{q_{1}}(z) \ldots f_{k}^{q_{k}}(z)+\varepsilon f_{1}^{q_{1}-1}(z) \ldots f_{k}^{q_{k}-1}(z) .
$$

Since $\lambda$ is a complete deformation of $D_{s}$, (3.26) may be induced from (3.25), modulo $\varepsilon^{2}$ and up to multiplication by a unit $u(z, \varepsilon) . u(z, \varepsilon)$ may be assumed to be of the form $u(z, \varepsilon)=1+\varepsilon u_{1}(z, \varepsilon)$, with $u_{1}(z, \varepsilon)$ holomorphic. Looking at the order of the zero of the coefficient of $\varepsilon$ at $z=0$, one sees that (3.26) may be induced from (3.25) only if $k=1$ and moreover $g_{1}(z, s(\varepsilon))=f_{1}(z)+\varepsilon u_{2}(z)$, modulo $\varepsilon^{2}$, with $u_{2}(0) \neq 0$.

We can see that, for small $\varepsilon \neq 0$ and small $z,\left\{z \mid g_{1}(z, s(\varepsilon))=0\right\}$ is nonsingular as follows. Multiplying by $1 / u_{2}(z)$, we may assume that $g_{1}(z, s(\varepsilon))$ is given by $F(z)+\varepsilon+\varepsilon^{2} G(z, \varepsilon)$ with $\{F(z)=0\}$ having a singularity only at the origin. For $i=1,2$, let the subscript $i$ denote the partial derivative with respect to $z_{1}$ and $z_{2}$ respectively. We want that $(0,0)$ is the only solution for small $z$ and $\varepsilon$ to the equations (3.27) and (3.28) below.

$$
\begin{gathered}
F(z)+\varepsilon+\varepsilon^{2} G(z, \varepsilon)=0, \\
F_{i}(z)+\varepsilon^{2} G_{i}(z, \varepsilon)=0, \quad i=1,2 .
\end{gathered}
$$

From (3.27), $\varepsilon=-F(z)[1+H(z)]$ with $H(z)$ holomorphic. Substituting into (3.28) gives

$$
F_{i}(z)+F^{2}(z) H^{(i)}(z)=0, \quad i=1,2,
$$

for holomorphic $H^{(i)}(z)$. Let $V$ be the set of $z$ satisfying (3.29). We need that $V=\{0\}$. If not, $V$ is one-dimensional. Let $\tau: T \rightarrow V$ be a resolution of a branch of $V$ at $0, z=z(t)$. From (3.29), $V \cap\{F(z)=0\}=0$. So $F$ is not identically 0 on $T$. An application of the chain rule shows that the order of the zero of $F(z(t))$ at $t=0$ is strictly greater than the order of the zero of $F_{i}(z(t))$ at $t=0$ for either $i=1$ or $i=2$. Then (3.29) cannot hold. Hence $V=\{0\}$ as desired. Hence the assumption that $x$ is a singular point of $W_{s}$ contradicts the fact that $\lambda_{1}$ is an equisingular deformation off $S_{1}$.

So to complete the proof of Theorem 3.5, we need that $\mathscr{D}_{s}$ is reduced. $\delta=\delta_{0}$ : $Q^{T_{0} \rightarrow H^{1}(D, \Phi)}$ is given as onto. Then as in the proof of [19, Theorem 3], $\delta_{s}$ :

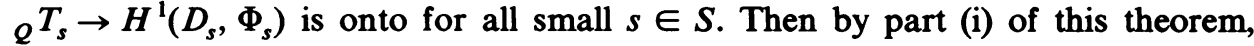
$H^{1}\left(D, \mathcal{T}_{s}\right)=0$. Let $g_{s}$ be the ideal sheaf of a component $C$ of $W_{s}$. If $q_{1}>1$, then $\sigma_{s} / \mathscr{G}_{s} \mathcal{T}_{s}$ is the sheaf of germs of sections of a line bundle over $C$ of Chern class $q_{1} C \cdot C$. Then $H^{1}\left(C, \mathscr{T}_{s} /{ }_{s} \mathscr{T}_{s}\right) \neq 0$. This contradicts $H^{1}\left(D_{s}, \mathscr{T}_{s}\right)=0$ and completes the proof of Theorem 3.5. 
We shall now give an example of a nonzero second obstruction to lifting a cycle. The example was suggested by work of Wahl [27]. Consider a rational singularity whose minimal resolution has the dual graph

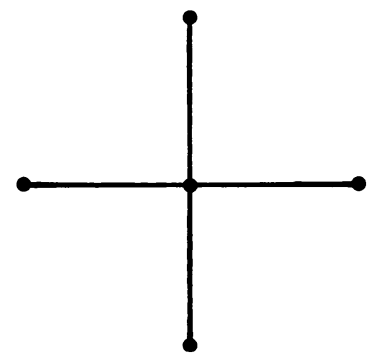

Let $E_{1}$ be the central vertex and $E_{2}, E_{3}, E_{4}, E_{5}$ the outer vertices. All $E_{i}$ are nonsingular rational curves. Let $E_{1} \cdot E_{1}=-3 ; E_{i} \cdot E_{i}=-2, i=2,3,4,5$. The fundamental cycle is given by $Z=2 E_{1}+E_{2}+E_{3}+E_{4}+E_{5}$. We may choose coordinates $(x, y)$ near $E_{1} \cap E_{2},\left(x_{1}, y_{1}\right)$ near $E_{2}-E_{1}$ and $\left(x_{2}, y_{2}\right)$ near $E_{1}-E_{2}$ with coordinate transitions $x_{1}=x y^{2}, y_{1}=1 / x, x_{2}=1 / x, y_{2}=x^{3} y$. Consider the following infinitesimally nontrivial deformation, with one-dimensional parameter $\varepsilon$ :

$$
\begin{array}{ll}
x_{1}=x y^{2}+2 \varepsilon y, & y_{1}=1 / x, \\
x_{1}=1 / x, & y_{2}=x^{3} y+\varepsilon x^{2} .
\end{array}
$$

The deformation is trivial on the other changes of coordinates. The cycle $Z$ may be lifted to first order by the defining equations

$x_{1}$ in $\left(x_{1}, y_{1}, \varepsilon\right)$ coordinates,

$x y^{2}+2 \varepsilon y$ in $(x, y, \varepsilon)$ coordinates,

$y_{2}^{2}$ in $\left(x_{2}, y_{2}, \varepsilon\right)$ coordinates away from $E_{3}, E_{4}$ and $E_{5}$.

$(3.1)_{1}$ between $(x, y, \varepsilon)$ and $\left(x_{2}, y_{2}, \varepsilon\right)$ coordinates is given by $y_{2}^{2}=x^{6} y^{2}+2 \varepsilon x^{5} y+$ $\varepsilon^{2} x^{4} \equiv x^{5}\left(x y^{2}+2 \varepsilon y\right)$. Then $\operatorname{cls}\left[x^{4}\right] \in H^{1}(Z, \Phi)$ is the second obstruction. Let 9 be the ideal sheaf of $E_{1}$. Let $\mathcal{T}$ be the sheaf of germs of infinitesimal deformations of $Z$. Then $\mathcal{T} / \mathcal{G} \mathcal{T}$ is isomorphic to the sheaf of germs of sections of a line bundle of Chern class $E_{1} \cdot Z=-2$ over $E_{1}$. Then via (2.9), the image of $\operatorname{cls}\left[x^{4}\right]$ in $H^{1}\left(E_{1}, \mathcal{T} / \mathcal{G} \mathcal{T}\right)$ is nonzero.

Observe that $Z$ is a candidate from [18, Proposition 2.3, p. 4] for weak lifting. Also, in the construction of Theorem 3.5, we are really concerned with higher obstructions in $H^{1}(Z, \Phi) / H^{1}(Z, \Re) \cdot \operatorname{cls}\left[x^{4}\right]$ is nonzero in $H^{1}(Z, \Phi) / H^{1}(Z, \Re)$.

TheOREM 3.6. Let $M$ be a strictly pseudoconvex two-dimensional manifold. Let $A=\cup A_{i}, 1<i<n$, be the decomposition of the exceptional set into irreducible components. Suppose that no $A_{i}$ is an exceptional curve of the first kind. Given any $A_{k}$, let $A^{\prime}=\cup A_{i}, i \neq k$. Then there exists a deformation $\omega: \operatorname{Nl} \rightarrow T$ of $M$ with $T$ smooth and one-dimensional such that for $t \neq 0, M_{t}$ has an exceptional set $A_{t}$ with exactly $n-1$ irreducible components, $A_{t, i}, 1<i<n$ and $i \neq k$, with $A_{t, i}$ homologous in $\mathfrak{N}$ to $A_{i}, i \neq k$. Moreover, $\omega$ induces a trivial deformation of the (reduced) analytic space $A^{\prime}$. 
Proof. Let $\tau: \mathcal{T} \rightarrow Q$ be the versal deformation of $M=N_{0}=\tau^{-1}(0)$ [19, Theorem 8]. Proposition 2.2 says that there is a maximal subspace $S$ of $Q$ to which $A^{\prime}$ weakly lifts. Also by Proposition $2.2, S$ is also maximal for all $q$ sufficiently near to 0 . Since $A^{\prime}$ is a reduced space, $\mathcal{T}$ has support only at the isolated singular points of $A^{\prime}$. Then $H^{1}(D, \mathcal{T})=0$. Let $\lambda: \mathscr{D} \rightarrow S$ be the complete deformation of $A^{\prime}$ from Theorem 3.5(iv).

$\lambda$ may be induced from the versal deformation of $A^{\prime}$ [8]. So there is a maximal submanifold $T_{1}$ of $S$ where $\lambda$ is the trivial deformation of $A^{\prime}$ near $0 . T_{1}$ is also maximal at all points $t \in T_{1}$.

By [18, Theorem 2.2, p. 4], off of a proper subvariety $V$ of $T_{1}, \tau$ induces an equisingular deformation of the full exceptional set. For $t \in T_{1}-V$, we shall show that the existence of an additional component $B_{t}$ of the exceptional set $A_{t}$, with $B_{t}$ not homologous to an $A_{i}, i \neq k$, leads to a contradiction. $B_{t}$ is not an exceptional curve of the first kind [1, Lemma 2.1, p. 334]. As in the proof of Theorem 2.8, we can find a special cover $\mathfrak{U}$ of $N_{t}=\tau^{-1}(t)$ and a cocycle $c$ with $[c] \in H^{1}\left(N_{t}, \Theta_{t}\right)$ such that the lifting of $B_{t}$ is obstructed in the direction of $c$. Moreover, $c$ will vanish near $A^{\prime}$. As in the proof of [19, Theorem 1], we may construct a deformation $\pi$ of $N_{t}$ in the direction of $c$ which induces the trivial deformation on $A^{\prime}$. Since $\tau$ is a complete deformation at $t\left[19\right.$, Theorem 8] and since $T_{1}$ is the maximal subspace of $Q$ near $t$ over which there is a trivial deformation of $A_{t}^{\prime}, \pi$ may be induced from $\tau$ restricted to $T_{1}$. But then $B_{t}$ cannot exist on the exceptional set over $T_{1}-V$.

To find the desired $T$ of the theorem, just take a 1-dimensional submanifold of $T_{1}$ such that $T \cap V=\{0\}$.

\section{REFERENCES}

1. M. Artin, Algebraic construction of Brieskorn's resolutions, J. Algebra 29 (1974), 330-348.

2. M. Artin and M. Schlessinger, Algebraic construction of Brieskorn's resolutions, preprint.

3. A. Douady, Le probleme des modules locaux pour les espaces $C$-analytiques compacts, Ann. Sci. École Norm. Sup. (4) 7 (1974), 569-602 (1975).

4. R. Elkik, Algébrisation du module formel d' une singularité isolée, Astérisque 16 (1974), 133-144.

5. W. Fischer and H. Grauert, Lokal-triviale Familien kompakter komplexer Mannigfaltigkeiten, Nachr. Akad. Wiss. Göttingen Math.-Phys. Kl. II 1965, 89-94.

6. H. Grauert, Über Modifikationen und exzeptionelle analytische Mengen, Math. Ann. 146 (1962), 331-368.

7. , Über die Deformation isolierter Singularitäten analytischer Mengen, Invent. Math. 15 (1972), 171-199.

8. _ Der Satz von Kuranishi für kompakte komplexe Räume, Invent. Math. 25 (1974), 107-142.

9. R. Gunning and R. Narasimhan, Immersion of open Riemann surfaces, Math. Ann. 174 (1967), 103-108.

10. R. Gunning and H. Rossi, Analytic functions of several complex variables, Prentice-Hall, Englewood Cliffs, N. J., 1965.

11. A. Kas and M. Schlessinger, On the versal deformation of a complex space with an isolated singularity, Math. Ann. 196 (1972), 23-29.

12. K. Kodaira, $A$ theorem of completeness of characteristic systems for analytic families of compact submanifolds of complex manifolds, Ann. of Math. 75 (1962), 146-162.

13. K. Kodaira and D. Spencer, $A$ theorem of completeness of characteristic systems of complete continuous systems, Amer. J. Math. 81 (1959), 577-500.

14. H. Laufer, On rational singularities, Amer. J. Math. 94 (1972), 597-608.

15. _ Deformations of resolutions of two-dimensional singularities, Rice Univ. Studies 59 (1973), 53-96. 
16. __ Taut two-dimensional singularities, Math. Ann. 205 (1973), 131-164.

17. ___ Ambient deformations for one-dimensional exceptional sets, 1978, preprint.

18. __ Ambient deformations for exceptional sets in two-manifolds, Invent. Math. 55 (1979), 1-36.

19. __ Versal deformations for two-dimensional pseudoconvex manifolds, Ann. Scuola Norm. Sup. Pisa Cl. Sci. (4) 7 (1980), 511-521.

20. _ On CP $\mathbf{P}^{1}$ as an exceptional set, Recent Developments in Several Complex Variables (J. Fornaess, Ed.), Ann. of Math. Studies No. 100, Princeton Univ. Press, Princeton, N. J., 1981, pp. 261-275.

21. D. Lê and C. Ramanujan, The invariance of Milnor's number implies the invariance of the topological type, Amer. J. Math. 98 (1976), 67-78.

22. G. Pourcin, Déformation de singularités isolées, Quelques Problèmes de Modules (Sem. Géométrie Analytique, Ecole Norm. Sup., Paris, 1971-1972), Astérisque, no. 16, Soc. Math. France, Paris, 1974, pp. 161-173.

23. O. Riemenschneider, Familien komplexer Räume mit streng pseudokonvexer spezieller Faser, Comment. Math. Helv. 51 (1976), 547-565.

24. Y.-T. Siu, Analytic sheaf cohomology groups of dimension $n$ of $n$-dimensional complex spaces, Trans. Amer. Math. Soc. 143 (1969), 77-94.

25. B. Teissier, Deformations à type topologique constant. I, Astérisque 16 (1974), 215-227.

26. J. Wahl, Equisingular deformations of normal surface singularities. I, Ann. of Math. 104 (1976), $325-356$.

27.

28. __ Simultaneous resolution and discriminantal loci, Duke Math. J. 46 (1979), 341-374.

29. H. Whitney, Tangents to an analytic variety, Ann. of Math. 81 (1965), 496-549.

Department of Mathematics, State University of New York, Stony BroOK, New York 11794 